\title{
Analytical Low-Thrust Satellite Maneuvers for Rapid Ground Target Revisit
}

\author{
Ciara N. McGrath ${ }^{1}$ and Malcolm Macdonald ${ }^{2}$ \\ University of Strathclyde, Glasgow, United Kingdom, G1 1XJ
}

This paper presents an analytical solution for a low-thrust maneuver to reduce the flyover time of a given terrestrial target. The work extends the general solution previously developed by the authors for a 3-phase spiral transfer that results in a change in the relative right ascension of the ascending node and argument of latitude of satellites in a constellation, by varying the orbital period and the $J_{2}$ effect experienced by each satellite. This work improves the accuracy of the existing method by including the periodic effects of $J_{2}$ in the analytical solution. Using these improved equations, a calculation of the flyover time of a given latitude can be determined, and the passes for which the target longitude is in view identified. Validation against a numerical orbit propagator shows the analytical method to accurately predict the sub-satellite point of the satellite to within $\pm 1^{\circ}$ of longitude after 15 days. A case study is performed showing that the method can successfully be used to reduce the time of flyover of Los Angeles from 14 days to just 1.97 days, with a change of velocity $(\Delta \mathrm{V})$ of $63 \mathrm{~m} / \mathrm{s}$. The full exploration of the solution space shows the problem to be highly complex, such that an increase in the $\Delta \mathrm{V}$ used for a maneuver will not necessarily reduce the time of flyover, potentially making optimization using a numerical solution challenging. It also shows that very similar flyover times can be achieved with very different $\Delta \mathrm{V}$ usage. As such, an overview of the solution space is extremely valuable in allowing an informed trade-off between the time of flyover and maneuver $\Delta \mathrm{V}$.

\section{Nomenclature}

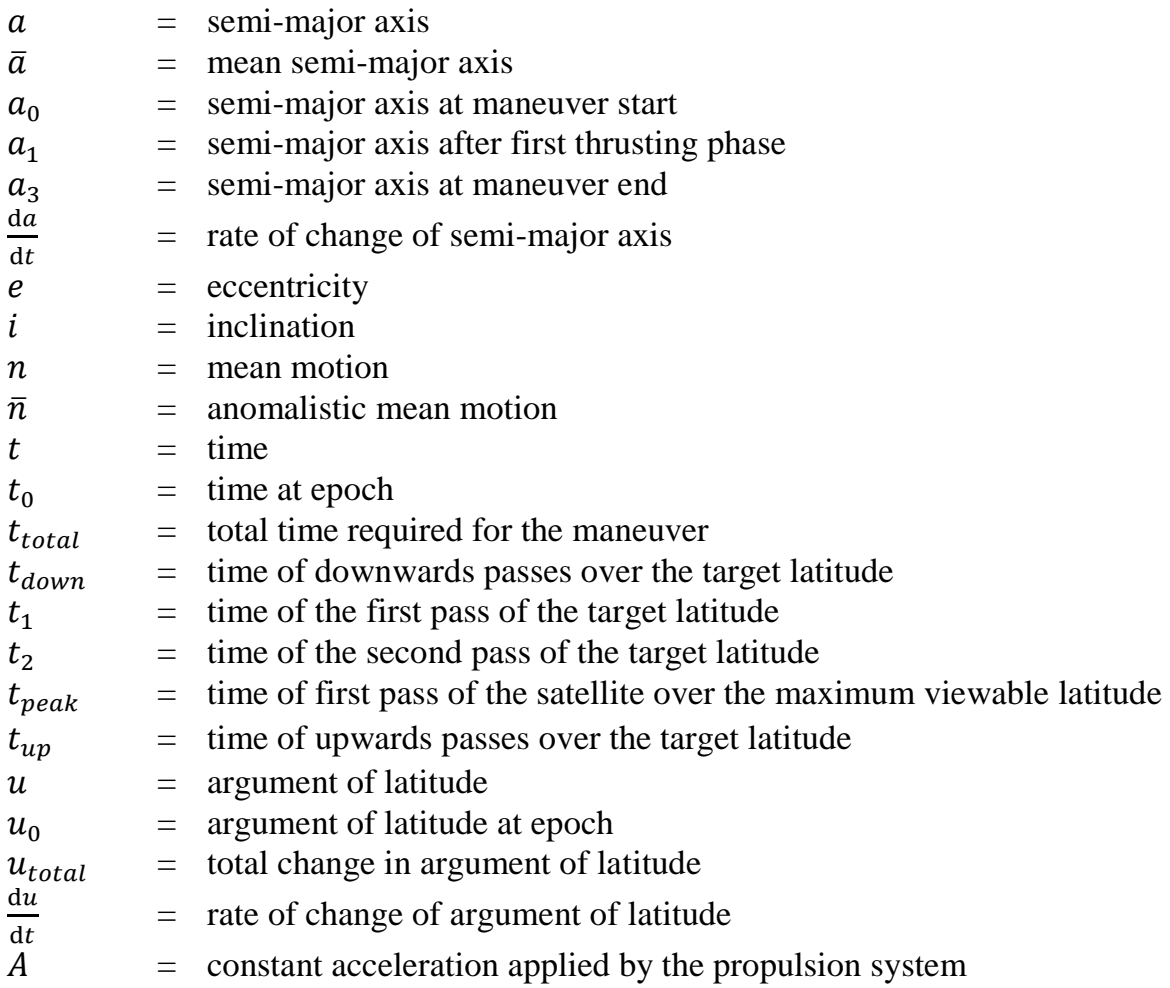

\footnotetext{
${ }^{1}$ Ph.D. Candidate, Department of Mechanical and Aerospace Engineering, James Weir Building $8^{\text {th }}$ Floor, 75 Montrose Street, Glasgow, G1 1XJ, AIAA Student Member.

${ }^{2}$ Reader, Department of Mechanical and Aerospace Engineering, James Weir Building $8{ }^{\text {th }}$ Floor, 75 Montrose Street, Glasgow, G1 1XJ, and AIAA Associate Fellow.
} 


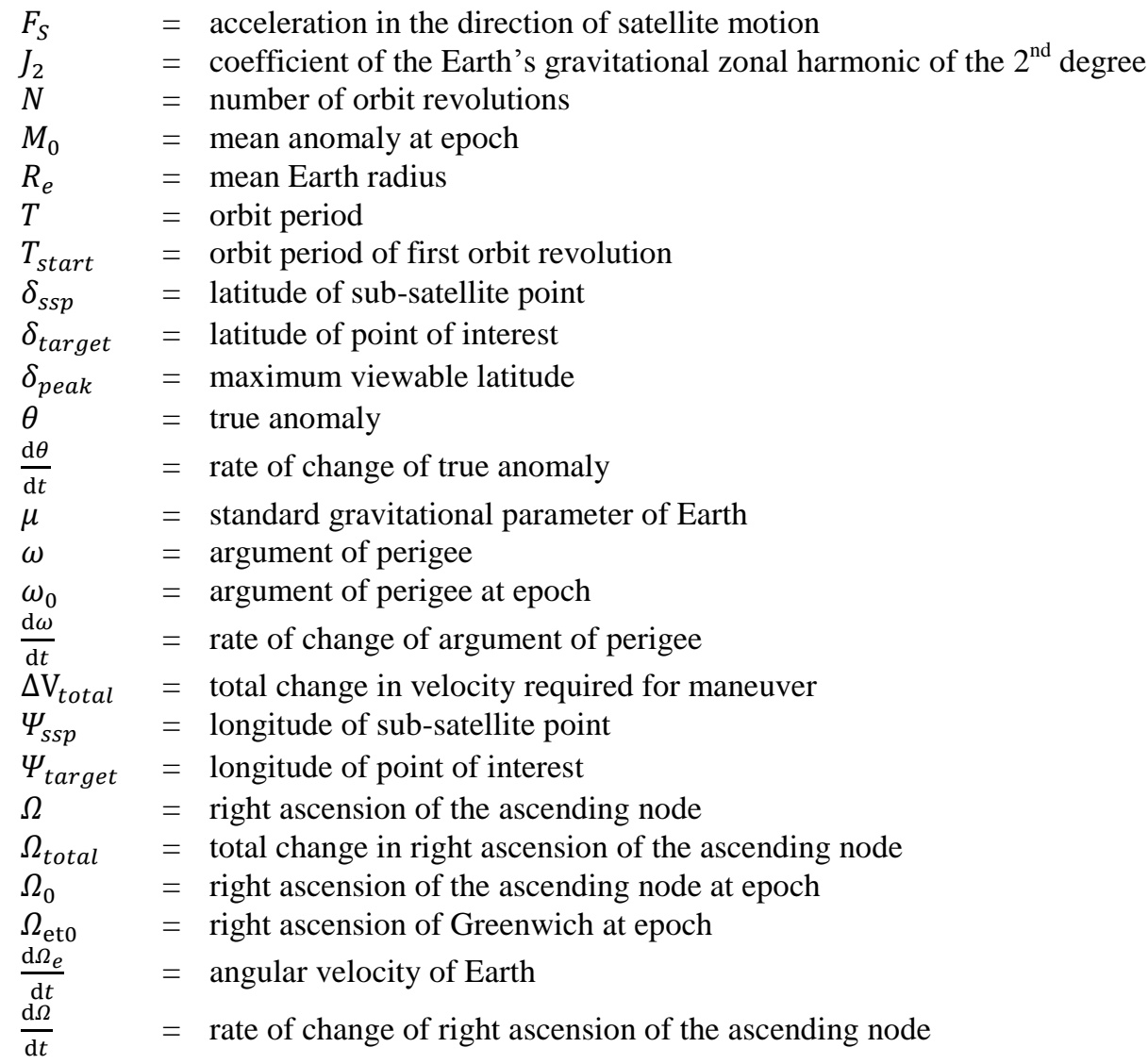

\section{Introduction}

$\mathrm{M}$

ORE than 100 satellites are currently in orbit performing Earth Observation (EO) missions, critical to supporting life on Earth. ${ }^{1}$ Weather monitoring, ${ }^{2}$ climate monitoring, ${ }^{3,4}$ Earth imaging, ${ }^{5}$ and disaster response $^{6}$ are all crucial services which make use of EO satellite data. Many of these applications require observations to be made of the entire Earth, utilizing multiple satellites arranged in a constellation. Particularly in the cases of weather monitoring, disaster response, and Earth imaging for commercial applications, rapid collection and dissemination of data from a given site is desirable. However, satellite orbits must be predefined before launch, and often are fixed early in the mission design. With limited on-board propellant available, most conventional satellite constellations remain static throughout the mission lifetime and are incapable of responding to a change in user needs. Although much work has been done in the area of Operationally Responsive Space (ORS), aiming to significantly reduce the time from mission conception to satellite launch and operation, current methods propose a timeline on the order of months, rather than weeks or days. ${ }^{7,8}$ As such, reconfigurable constellations capable of providing coverage of a given target, as required, would be extremely useful, enabling products and services to respond to real-time needs or market demands. This would significantly increase the commercial potential of such a system when compared to a static constellation, and could allow for unique mission objectives and requirements to be met.

Guelman and Kogan proposed the use of electric propulsion as a method of satellite reconnaissance (i.e. providing overflight of multiple successive ground targets), in 1999. ${ }^{9}$ They determined the problem to be a discrete optimization problem and thus used a simulated annealing method to identify the minimum fuel trajectory that would satisfy the requirement to pass over a set of ground targets in a given time. Jean and de Lafontaine went on to develop an autonomous control law capable of fulfilling such a mission. ${ }^{10}$ Work has also been done on optimizing the orbit used to overfly the targets. Abdelkhalik tackles the problem by using a genetic algorithm to select a natural orbit which will overfly as many ground targets as possible from a given set, without the need for a propulsion system. ${ }^{11,12}$ A special case of the reconnaissance mission is one in which rapid revisit of a specific target is required. Kim addresses this by using a genetic algorithm to define a new temporary orbit which would minimize the average revisit time of a given target. ${ }^{13}$ Another aspect of the problem is the optimal reassignment of each satellite in a constellation. De Weck used an auction algorithm to reliably determine the optimal assignment of satellites when reconfiguring a constellation. ${ }^{14}$ His focus is on the staged deployment of satellite constellations, but a similar reassignment problem exists in all cases of constellation reconfiguration. 
Although most studies of maneuverable satellite missions have focused on low-thrust trajectories, Zhu proposes the use of high-thrust maneuvers based on a Hohmann transfer to achieve flyover of a given ground target. ${ }^{15}$ Multiple objectives are considered, including maximizing coverage time of the target and minimizing the fuel required for the orbit transfer. Combining these into a fitness function, Zhu uses particle swarm optimization and differential evolution to select a satellite from an available set and to optimize the maneuver trajectory. However, Zhu notes that an optimal solution cannot be guaranteed. This is a weakness of the numerical optimizers that have mainly been used to tackle this problem to date, for both low- and high-thrust scenarios, as they do not provide a complete view of the solution space.

There have been some recent attempts to solve the reconnaissance problem analytically. Zhang's work provides approximate, semi-analytical solutions to the problem using high-thrust propulsion. ${ }^{16,17}$ His method, based on Kepler's equations and considering up to four impulsive maneuvers, allows for the minimum energy trajectory to be found. The results are found to be sub-optimal compared with a numerical solver, but Zhang highlights that they could provide an initial guess for higher precision models and may be useful in cases where fast computational speed is desired.

For the case of low-thrust propulsion, Co's work in recent years has developed a control algorithm which allows a single satellite to pass over a given target using electric propulsion. ${ }^{18-20}$ Co uses the difference in time of flyover between the maneuvering satellite and a non-maneuvering reference satellite as a metric, and can solve for this using a single equation. Co's algorithm propagates the initial orbit for a given time and identifies close passes to the target. These are then ordered in terms of the soonest encounter, if the fastest over-flight solution is desired, or by order of closest pass if the minimum fuel solution is desired. Discarding those which are infeasible, the fastest, or minimum energy, feasible solution can be found. Being based on a straightforward, analytical expression, Co's solution allows for analysis of the possible reach of a maneuver of this type, and the impact of the initial orbit parameters on the maneuver efficiency. However, it does not provide a full overview of the solution space and instead solves for a single solution, optimizing for either time, fuel, or a cost function combining the two.

The work presented here aims to complement the above research by developing a fully analytical solution describing a low-thrust, in-plane satellite manoeuver to reduce the revisit time to a specific target. This is a special case of the reconnaissance problem, and could be extended to consider flyover of multiple terrestrial targets. This analytical solution is developed based on previous work by the authors, which analytically describes the reconfiguration of a satellite constellation. ${ }^{21,22}$ These solutions will provide an overview of the entire solution space, enabling mission designers to understand the capabilities and limitations of such a maneuver and inform mission design and operation.

\section{Method}

Previous work by the authors has shown that by making use of low-thrust propulsion and exploiting the Earth's natural perturbing forces, it is possible to analytically describe the reconfiguration of a satellite constellation, achieving a desired separation of both right ascension of the ascending node (RAAN) and rate of change of the argument of latitude (AoL) between satellites. ${ }^{21}$ The technique requires that a satellite raise or lower its semi-major axis, creating a difference in the $J_{2}$ effect experienced in relation to other satellites in the constellation. The general solution allows for the orbit altitude to be raised or lowered beyond the target altitude in order to achieve a faster plane change maneuver if required, as shown in Fig. 1 (a). This method has been successfully used to define a low-cost deployment strategy for satellite constellations. ${ }^{22}$ The deployment maneuvers were optimized and showed a significant reduction in cost when compared with traditional launch methods, particularly for constellations with a large number of orbital planes.

This paper presents the use of the same technique to enable faster revisit times of points of interest (POIs) on the ground. In this case it is assumed that the satellite must begin and end the maneuver at the same altitude, to enable consistent observation of the Earth using the on-board instruments. However, the method could similarly be used to finish the maneuver at a different altitude. It is also assumed that the initial maneuver phase will lower the satellite altitude, as previous work found this to be the most efficient maneuver style, but the alternative of initially increasing the satellite altitude could also be considered. ${ }^{21}$ The chosen maneuver style is illustrated in Fig. 1 (b). 


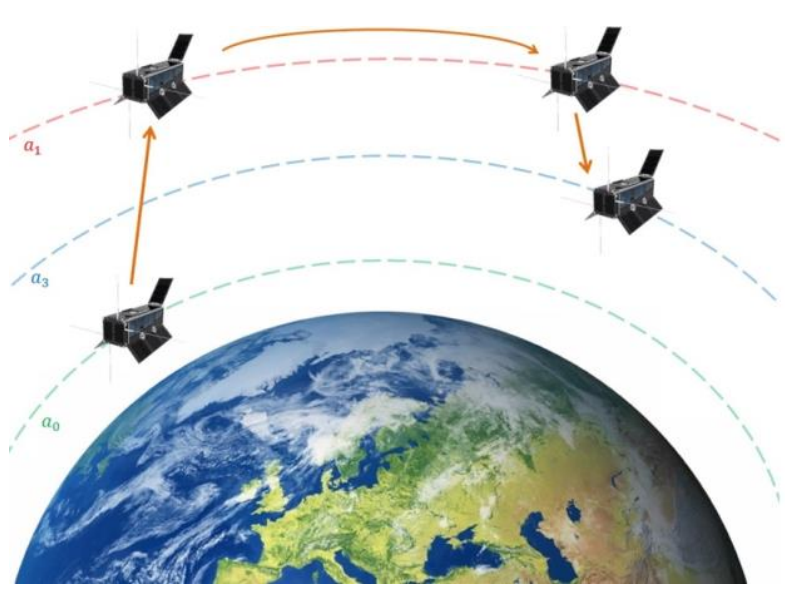

(a)

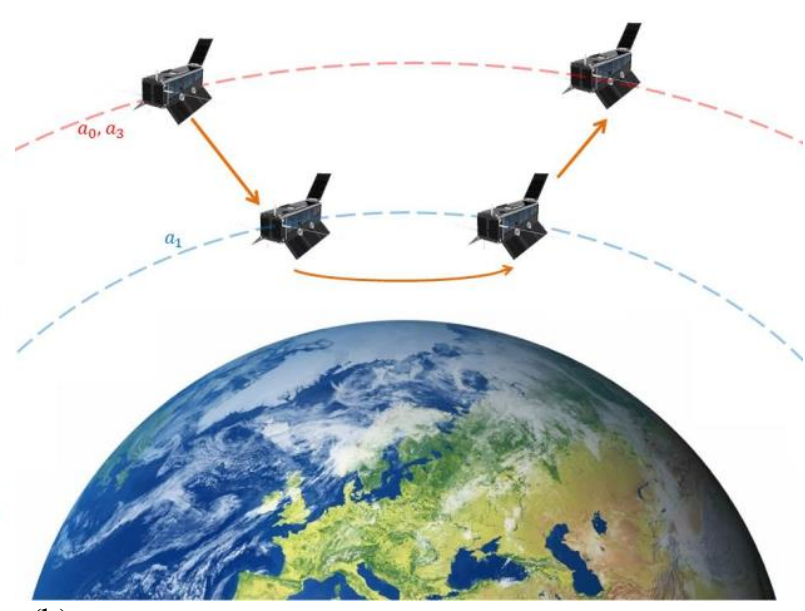

(b)

Figure 1. (a) General 3-Phase reconfiguration maneuver and (b) 3-Phase maneuver returning to initial altitude as used herein. Initial orbit $\left(a_{0}\right)$, intermediate orbit $\left(a_{1}\right)$ and final orbit $\left(a_{3}\right)$ are marked by dashed lines.

\section{A. Analytical description of maneuvers}

The Gauss version of the Lagrange planetary equations have been used to derive analytical expressions describing the total change in RAAN and AoL achievable using this style of maneuver, considering the effects of Earth oblateness up to the order of $J_{2}$. Only circular orbits are considered and atmospheric drag, solar radiation pressure, and third body perturbations are not included. In order to simplify the expressions, the equations previously derived discounted the change in argument of perigee and mean anomaly, as well as the periodic change in semi-major axis, caused by the Earth's oblateness. Although this was acceptable in previous work where the focus was on separation of the satellites through a desired RAAN, significant errors arise in the AoL with this simplification, on the order of $>1^{\circ}$ per day. To ensure that the ground track is sufficiently accurate for analysis of flyover times of POIs, it is necessary to include these effects.

1. Analytical expression for change in AoL using anomalistic mean motion and average semi-major axis

Considering the effect of the oblate Earth, the change in AoL is given as

$$
\frac{\mathrm{d} u}{\mathrm{~d} t}=\frac{\mathrm{d} \theta}{\mathrm{d} t}+\frac{\mathrm{d} \omega}{\mathrm{d} t}
$$

where the rate of change of true anomaly is given by

$$
\frac{\mathrm{d} \theta}{\mathrm{d} t}=\bar{n}=n\left(\frac{3}{2}\left(1-e^{2}\right)^{-3 / 2} J_{2}\left(1-\frac{3 \sin ^{2}(i)}{2}\right)\left(\frac{R_{e}}{\bar{a}}\right)^{2}+1\right)
$$

and the rate of change of argument of perigee is given by

$$
\frac{\mathrm{d} \omega}{\mathrm{d} t}=\bar{n} \frac{3}{2} J_{2}\left(2-\frac{5 \sin ^{2}(i)}{2}\right)\left(\frac{R_{e}}{\bar{a}}\right)^{2}\left(1-e^{2}\right)^{-2} .
$$

Here, the unperturbed mean motion $n$ is defined as

$$
n=\sqrt{\frac{\mu}{\bar{a}^{3}}}
$$

and the average semi-major axis, accounting for the periodic oscillations caused by the oblate Earth, $\bar{a},{ }^{17,23,24}$ is given by

$$
\bar{a}=a_{0}-\frac{3 J_{2} R_{e}^{2}}{2 a_{0}} \sin ^{2}(i) \cos \left(2\left(M_{0}+\omega_{0}\right)\right)
$$


Knowing that

$$
M_{0}+\omega_{0}=u_{0}
$$

and making the assumption that each maneuver phase starts at a known semi-major axis $a_{0}$ with $u_{0}=0$, simplifies the equations further, allowing Eq. (1) to be integrated over the drift phase for the case where eccentricity $e=0$ and the semi-major axis remains constant. This gives an analytical expression for $u=f(t)$.

Considering the anomalistic mean motion, the equation for the rate of change of semi-major axis as a function of time is

$$
\frac{\mathrm{d} a}{\mathrm{~d} t}=\frac{2 F_{S}}{\bar{n}}
$$

where $F_{S}$ is the acceleration in the direction of satellite motion. This cannot be easily integrated when using the mean semi-major axis, and so the standard semi-major axis is used here as an approximation. Assuming that for electric propulsion the change in mass of the satellite is minimal, the thrust applied can be assumed to be of constant acceleration magnitude and is represented by $A$, where a positive value corresponds to acceleration in the direction of satellite motion, and a negative value corresponds to acceleration in the opposite direction. This gives

$$
\frac{\mathrm{d} a}{\mathrm{~d} t}=\frac{2 A}{\sqrt{\frac{\mu}{a^{3}}}\left(\frac{3 J_{2} R_{e}^{2}\left(1-\frac{3 \sin ^{2}(i)}{2}\right)}{2 a^{2}}+1\right)}
$$

for circular orbits with only in-plane thrust. The use of the standard semi-major axis rather than the average value introduces negligible errors when compared with a numerical simulation, and allows for a fully analytical expression to be used. Integrating this over the maneuver phases, an expression is derived for $t=f(a)$. Using this, an equation for the rate of change of AoL throughout the maneuver phase can be calculated as a function of the semi-major axis with

$$
\frac{\mathrm{d} u}{\mathrm{~d} a}=\frac{\mathrm{d} u}{\mathrm{~d} t} \frac{\mathrm{d} t}{\mathrm{~d} a}
$$

Using Eq. (1) and Eq. (8), and thereby integrating Eq. (9) over the maneuver phases produces an expression for $u=f(a)$.

Combining the derived equations to account for an initial spiral thrust phase, a coast phase and a second thrust phase, and knowing that the intermediate semi-major axis can be calculated as a function of the total change in velocity, $\Delta \mathrm{V}_{\text {total }}$, with

$$
a_{1}=\frac{4 a_{0} \mu}{a_{0}\left(2 \sqrt{\frac{\mu}{a_{0}}}+\sqrt{\frac{\mu}{a_{3}}}+\Delta \mathrm{V}_{\text {total }}\right)\left(\sqrt{\frac{\mu}{a_{3}}}+\Delta \mathrm{V}_{\text {total }}\right)+\mu}
$$

a single equation can be derived for $u_{\text {total }}=f\left(t_{\text {total }}, \Delta V_{\text {total }}\right)$.

2. Analytical expression for change in RAAN using anomalistic mean motion and average semi-major axis

Similar to the equations for AoL, the expressions for the change in RAAN can be improved using the anomalistic mean motion and the mean semi-major axis. For the drift phase, assuming a circular orbit and a constant semi-major axis, the rate of change of RAAN can be expressed as

$$
\frac{\mathrm{d} \Omega}{\mathrm{d} t}=-\frac{3 J_{2} \bar{n} R_{e}^{2} \cos (i)}{2 \bar{a}^{2}} \text {. }
$$

Integrating this over the drift phase gives an equation for $\Omega=f(t)$.

Making use of Eq. (8) and Eq. (11), an equation describing the rate of change of RAAN during the maneuver phases as a function of the changing semi-major axis can be defined as 


$$
\frac{\mathrm{d} \Omega}{\mathrm{d} a}=\frac{\mathrm{d} \Omega}{\mathrm{d} t} \frac{\mathrm{d} t}{\mathrm{~d} a}
$$

Integrating this over the maneuver gives an expression for $\Omega=f(a)$ and, as in the case of the AoL, combining the derived equations provides a single analytical expression giving $\Omega_{\text {total }}=f\left(t_{\text {total }}, \Delta V_{\text {total }}\right)$.

\section{B. Relating the change in RAAN and AoL to changes in latitude and longitude}

Using the equations for change in AoL and RAAN derived in section II.A, it is possible to analytically describe the position of the satellite post-maneuver in terms of the orbital elements. However, in order to target a specific POI on the ground, it is necessary to link these changes in orbital elements to changes in the ground track.

From spherical geometry the latitude of the sub-satellite point (SSP) can be calculated from the orbital elements using

$$
\delta_{s s p}=\sin ^{-1}\left(\sin (i) \sin \left(u_{\text {total }}\right)\right)
$$

and the longitude of the SSP can be calculated by

$$
\Psi_{s S p}=\tan ^{-1}\left(\frac{\cos (i) \sin \left(u_{\text {total }}\right)}{\cos \left(u_{\text {total }}\right)}\right)-\frac{\mathrm{d} \Omega_{e}}{\mathrm{~d} t} t_{\text {total }}+\Omega_{\text {total }}+\Omega_{0}-\Omega_{\text {et } 0}
$$

where $\Omega_{\text {eto }}$ is the right ascension of Greenwich at epoch and $\frac{\mathrm{d} \Omega_{e}}{\mathrm{~d} t}$ is the angular rotation rate of the Earth. ${ }^{5,24}$ Note that it is necessary to include the inverse tangent function in this form to allow for the quadrant of the angle to be correctly determined. Combining Eq. (13) and Eq. (14) with those derived in section II.A, gives expressions for $\delta_{s s p}=f\left(t_{\text {total }}, \Delta V_{\text {total }}\right)$ and $\Psi_{s s p}=f\left(t_{\text {total }}, \Delta V_{\text {total }}\right)$.

\section{Analysis}

\section{A. Overview of the solution space}

The orbit of the International Space Station (ISS) is selected for this analysis. These orbit parameters are laid out in Table 2. The acceleration of the electric propulsion system $A$ given here is consistent with that used by Co in his previous work. ${ }^{18-20}$ Table 1 defines the standard orbital parameters used for the analysis.

The ground target selected for revisit is Los Angeles, California. Earthquakes in Los Angeles are common occurrences, but while most are relatively small, a large magnitude earthquake could cause significant damage and even loss of life. In the aftermath of such an event, rapidly available satellite data would be a vital asset for response teams on the ground, and a satellite capable of targeting the region a number of times in short succession could be extremely valuable.

Table 1. Orbital Constants.

\begin{tabular}{lccc}
\hline \multicolumn{1}{c}{ Parameter } & Symbol & Value & Units \\
\hline Gravitational Parameter & $\mu$ & $3.986 \mathrm{E} 14$ & $\mathrm{~m}^{3} / \mathrm{s}^{2}$ \\
Radius of Earth & $\mathrm{R}_{\mathrm{e}}$ & $6.371 \mathrm{E} 3$ & $\mathrm{~km}$ \\
$\mathrm{~J}_{2}$ Parameter & $\mathrm{J}_{2}$ & $1.0827 \mathrm{E}-3$ & - \\
Angular velocity of Earth & $\omega_{e}$ & $7.2921 \mathrm{E}-5$ & $\mathrm{rad} / \mathrm{s}$ \\
\hline
\end{tabular}


Table 2. Mission Parameters.

\begin{tabular}{lcll}
\hline Parameter & Symbol & Value & Units \\
\hline Propulsion acceleration & $A$ & \pm 0.001 & $\mathrm{~m} / \mathrm{s}^{2}$ \\
Inclination & $i$ & 51.6431 & $\mathrm{deg}$ \\
Initial semi-major axis & $a_{0}$ & 6773 & $\mathrm{~km}$ \\
Final semi-major axis & $a_{3}$ & 6773 & $\mathrm{~km}$ \\
Initial AoL & $u_{0}$ & 0 & $\mathrm{rad}$ \\
Initial RAAN & $\Omega_{0}$ & 0 & $\mathrm{rad}$ \\
Latitude of POI & $\delta_{\text {target }}$ & 34.0522 & $\mathrm{deg}$ \\
Longitude of POI & $\Psi_{\text {target }}$ & -118.2437 & $\mathrm{deg}$ \\
Epoch & - & $01 \mathrm{Jan} 199000: 00: 00.0000$ & - \\
Right ascension of Greenwich at epoch & $\Omega_{\text {et0 }}$ & 100.38641 & $\mathrm{deg}$ \\
\hline
\end{tabular}

Plotting the expressions for the latitude and longitude of the sub-satellite point as a function of maneuver time and $\Delta V_{\text {total }}$, gives the surfaces shown in Fig. 2 and Fig. 3 respectively. It is clear from these graphs that it is possible to co-ordinate a flyover of any latitude for which $-i<\delta_{\text {target }}<i$, and for all longitudes, given the correct combination of $\Delta V_{\text {total }}$ and $t_{\text {total }}$. However the solution space is extremely complex.

Providing a value for $\Delta \mathrm{V}_{\text {total }}$ allows the latitude and longitude to be plotted as a function of time only. Figure 4 (a) and (b) show this for the case where $\Delta \mathrm{V}_{\text {total }}=100 \mathrm{~m} / \mathrm{s}$.

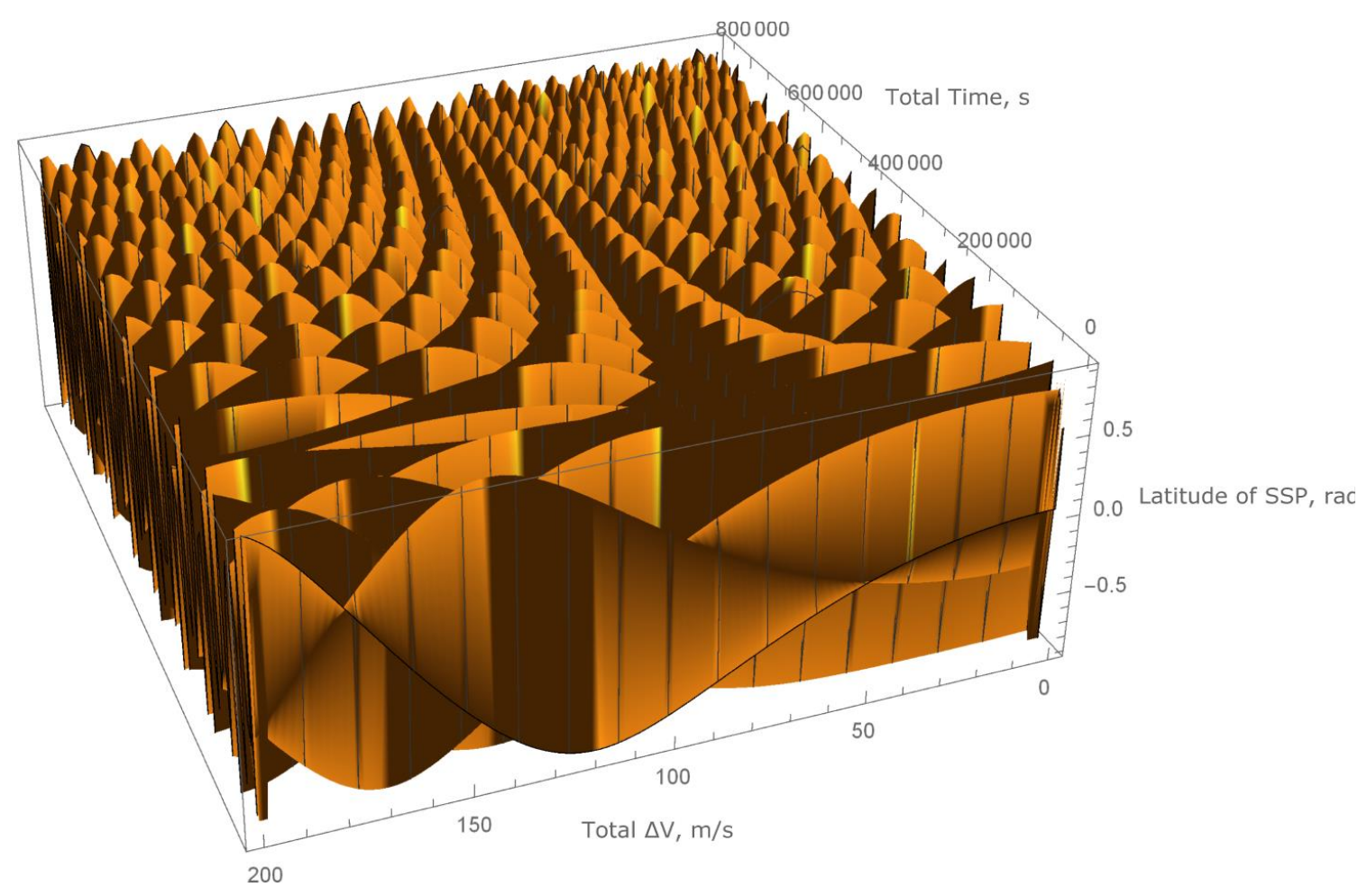

Figure 2. Latitude in radians plotted for $\mathbf{1 0}$ days as a function of $\Delta V_{\text {total }}$ and maneuver time. 


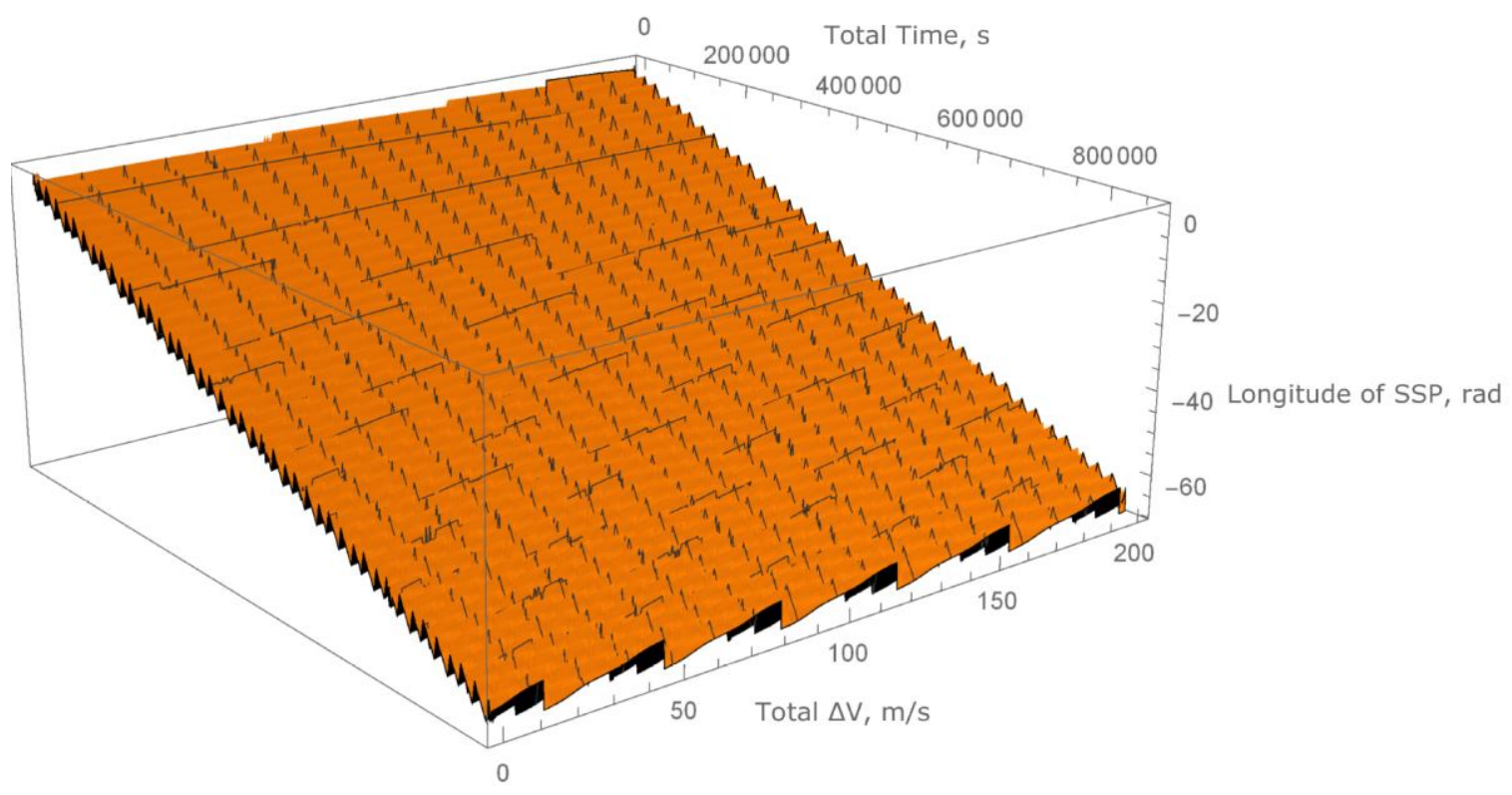

Figure 3. Longitude in radians plotted for 10 days as a function of $\Delta V_{\text {total }}$ and maneuver time

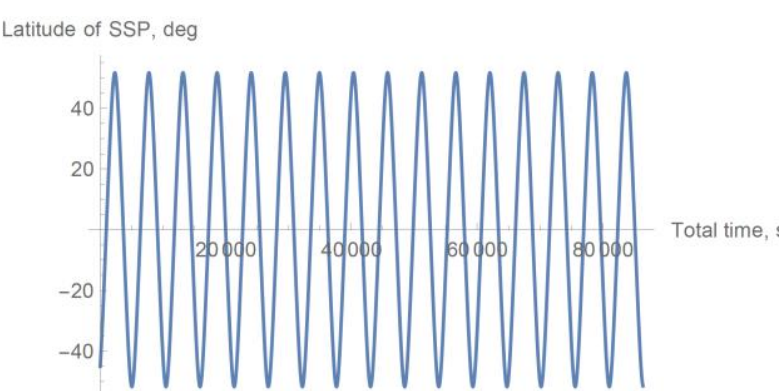

(a)

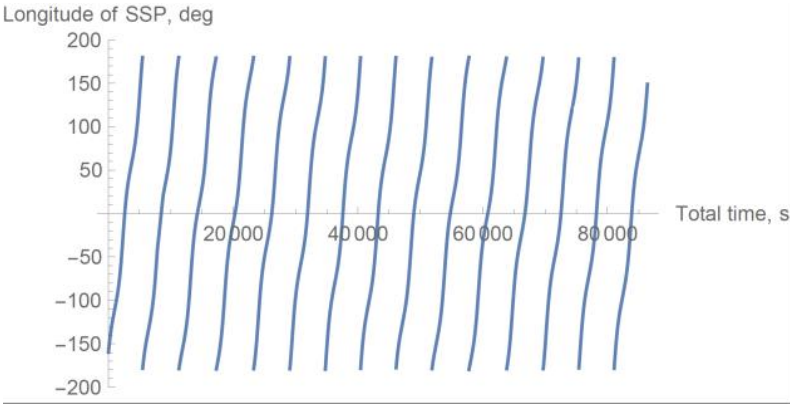

(b)

Figure 4. (a) Latitude and (b) longitude in degrees plotted for 1 day, for a maneuver $\Delta V_{\text {total }}$ of $100 \mathrm{~m} / \mathrm{s}$.

\section{B. Solving for flyover time of a given target}

The expression derived in section II.B above for $\Psi_{s s p}=f\left(t_{\text {total }}, \Delta V_{\text {total }}\right)$ cannot explicitely be solved for $t_{\text {total }}$ or $\Delta \mathrm{V}_{\text {total }}$ as the equation is implicit in both. The expression for $\delta_{s s p}=f\left(t_{\text {total }}, \Delta V_{\text {total }}\right)$ can be solved for $t_{\text {total }}$, but due to the nature of the trigonometric functions only the solution for the first pass of a given latitude can be found. However, it can be seen in Fig. 4 (a) that for a given $\Delta \mathrm{V}_{\text {total }}$ value, the time of flyover of any latitude is periodic. This is because the analytical method used to describe the maneuver creates an artificially longer first orbit period to account for the effect of the maneuver. All following orbit periods are then constant and are equivalent to the orbit period at the drift altitude $\left(a_{1}\right)$.

In order to find the times at which the satellite will pass over the POI, it is first necessary to find all the times at which the satellite passes the target latitude. Solving for the corresponding longitude at each of these times then allows those passes which are over the POI to be identified. This needs to be done for both the upwards and downwards passes of the target latitude.

To find the period of the first lengthier orbit period, the equation for $u_{\text {total }}=f\left(t_{\text {total }}\right)$ is rearranged to solve for $T_{\text {start }}$, when $u_{\text {total }}=2 \pi$. If $\Delta \mathrm{V}_{\text {total }}=0$ then this will just be the standard orbit period. To determine the periods of the following revolutions, the time required for two revolutions, i.e. $u_{\text {total }}=4 \pi$, is calculated and the time of the first period subtracted. 
The time of the first pass over the target latitude can be simply calculated by $t_{1}=f\left(\delta_{\text {target }}\right)$. Assuming this first pass is an upwards pass, a general expression for the time at which all upwards passes of the latitude of interest will occur can then be written as

$$
t_{\text {up }}=N T+T_{\text {start }}
$$

where $N \in \mathbb{Z}$ and expresses the number of orbit revolutions passed. An expression for the downwards passes can then be written as

$$
t_{\text {down }}=N T+T_{\text {start }}-t_{1}+t_{2}
$$

where

$$
t_{2}=\left(t_{\text {peak }}-t_{1}\right)+t_{\text {peak }}
$$

and $t_{\text {peak }}$ corresponds to the time at which the satellite passes the highest possible latitude (i.e. $\delta_{\text {peak }}=i$ ).

If the first pass is a downwards pass then the following expressions should be used

$$
\begin{gathered}
t_{\text {up }}=T_{\text {start }}-\left(t_{2}-t_{1}\right)+N T \\
t_{\text {down }}=N T+T_{\text {start }} .
\end{gathered}
$$

\section{Validation}

Validation of the analytical method described in section III was done against a numerical simulator which propagates the position of the spacecraft using a set of modified equinoctial elements ${ }^{25}$ using an explicit variable step size Runge Kutta (4,5) formula, the Dormand-Prince pair. ${ }^{26}$ This numerical simulation includes only perturbations due to Earth oblateness to the order of $J_{2}$. The acceleration control law applies a constant acceleration of $-A$ to lower the satellite altitude for a duration of $\frac{\Delta \mathrm{V}_{\text {total }}}{2|A|}$, and then a constant acceleration of $A$ for a duration of $\frac{\Delta \mathrm{V}_{\text {total }}}{2|A|}$ to return the satellite to the initial altitude after the drift period. The results of the analytical method were used to define the thrusting time and drift time of the maneuver performed using the numerical simulator.

\section{A. Validation of the method for a non-maneuvering satellite}

\section{Analytical Method}

Considering a non-maneuvering satellite, starting with the initial conditions laid out in Table 1 and Table 2, the analytical method described in section III.B can be used to plot each satellite pass over the target latitude, and the corresponding longitude. Figure 5 shows this for the first 100 upwards and downwards passes. The grey horizontal line indicates the target longitude. Figure 6 shows for the first 300 upwards and downwards passes, the distance between the sub-satellite point and the point of interest as calculated using the haversine formula. ${ }^{5}$ The red line indicates the distance below which the point of interest is visible to the satellite. This is calculated based on the satellite swath width, which in this case is taken as $184 \mathrm{~km}$. This corresponds to $\pm 1^{\circ}$ of the target longitude at this latitude.

In Fig. 6 an upwards pass over Los Angeles after 2 revolutions is visible, marked with the letter 'A'. 'B' marks a downwards pass after 22 revolutions, which corresponds to 1.43 days from epoch. Pass ' $\mathrm{C}$ ' occurs on revolution 237 at 15.21 days from epoch. 


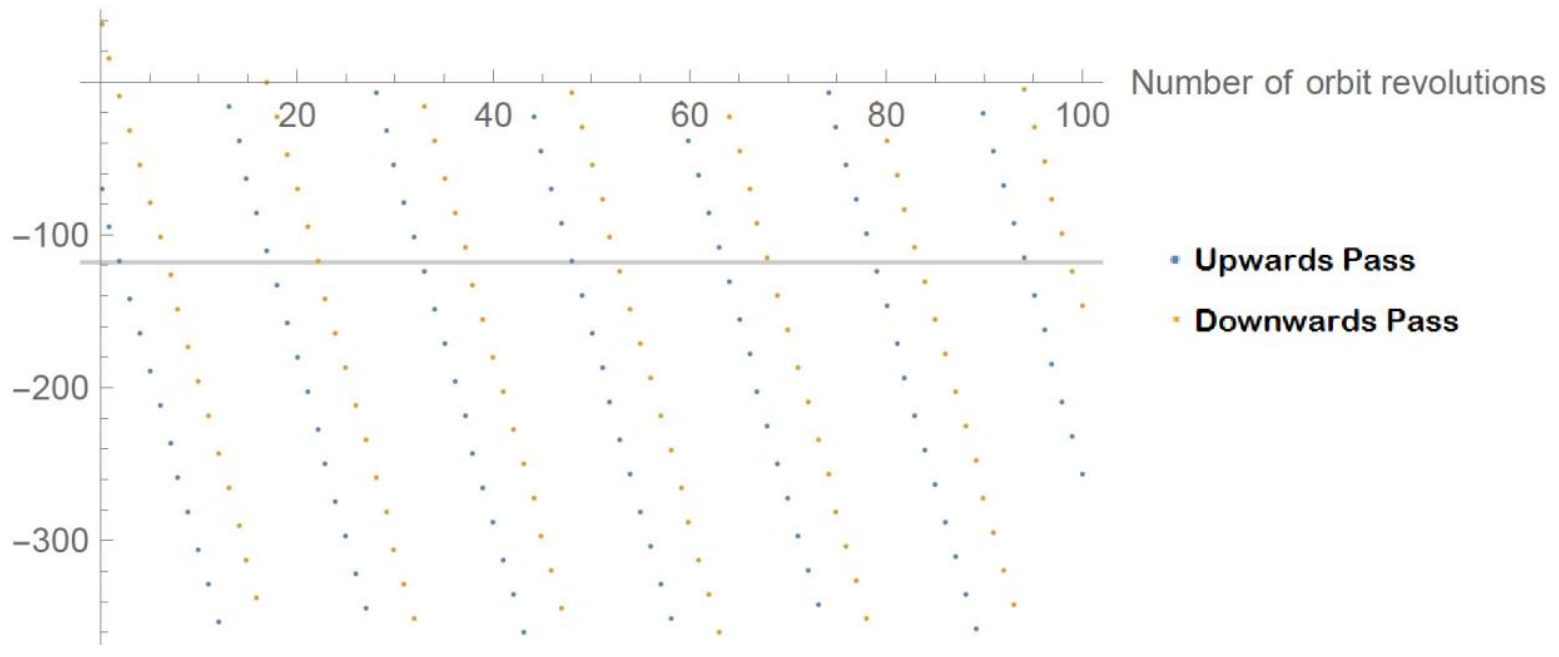

Figure 5. The longitude of the SSP for the first 100 passes over the target latitude with a non-maneuvering satellite. Blue dots mark an upwards pass and orange dots mark a downwards pass. The horizontal grey line indicates the target longitide.

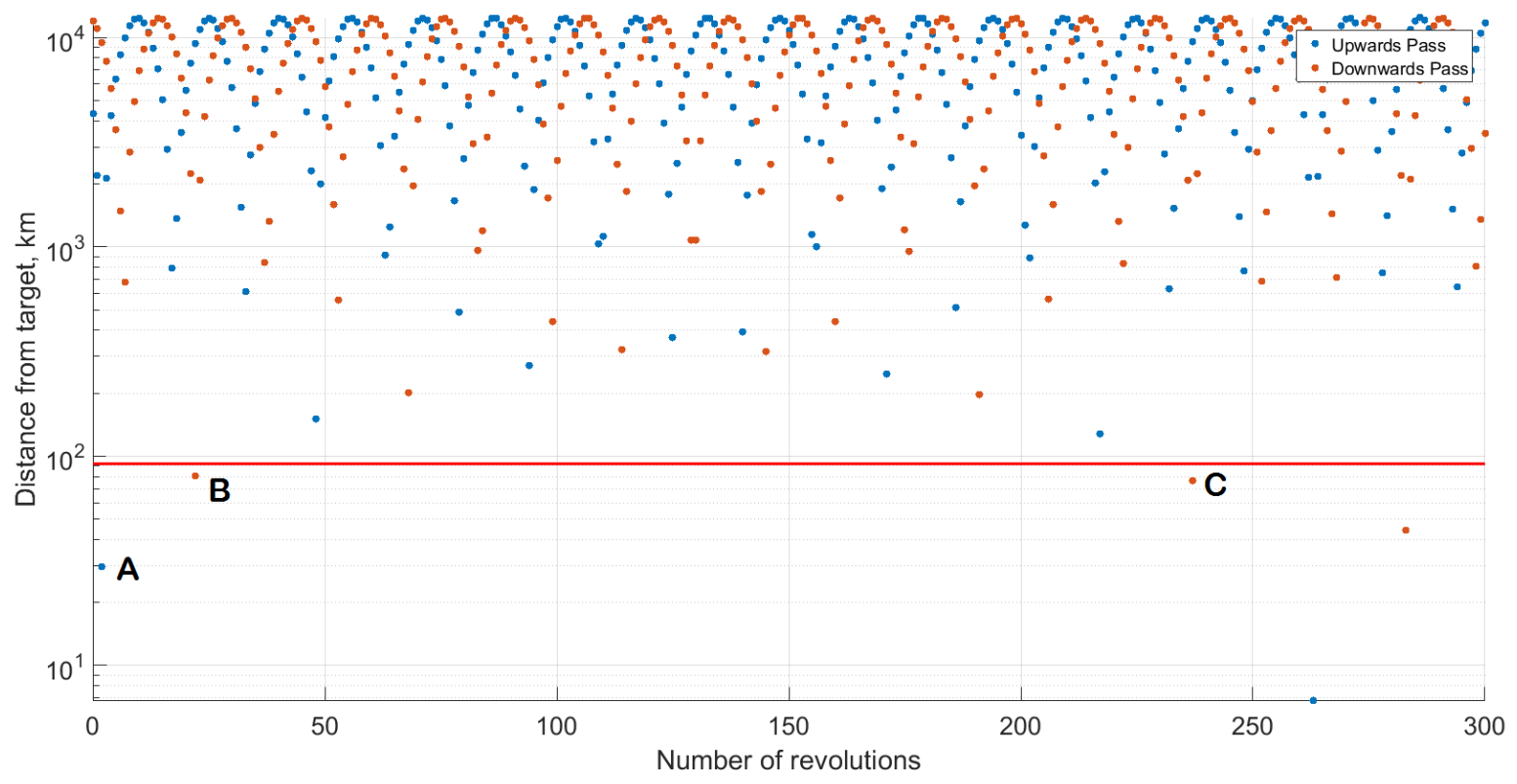

Figure 6. The distance from the SSP to the POI, plotted for the first 300 revolutions for a nonmaneuvering satellite. Blue dots mark an upwards pass and red dots mark a downwards pass. The horizontal red line indicates the distance below which the POI will be visible. A, B and C mark the first, second and third passes for which the POI is visible.

\section{Numerical Simulation}

Using the numerical simulator, the satellite orbit is propagated for 15.21 days, and the latitude and longitude of the sub-satellite point is calculated at all time-steps. Figure 7 shows the ground track produced in this manner. The blue dot indicates the satellite position at the beginning of the simulation and the green dot shows the position of the satellite over Los Angeles at the end of the simulation. Figure 8 shows the altitude of the satellite throughout the simulation showing that it varies very little over the 15 day simulation. Figure 9 
shows the RAAN and AoL of the satellite throughout the simulation. Figure 10 shows the distance of the subsatellite point from the target POI as calculated using the haversine formula at each time step of the numerical simulation. The red line indicates the distance below which the POI will be visible to the satellite. The first two peaks correspond to passes A and B predicted by the analytical method, and the third peak at 15.21 days corresponds to pass $\mathrm{C}$. It can be seen that, as predicted by the analytical method, there is no other pass during which Los Angeles will be in view for this given swath, even though it may pass closely at times. For all three of these passes the latitude and longitude of the sub-satellite point calculated using the analytical method matches the numerical simulation to within $\pm 1^{\circ}$.

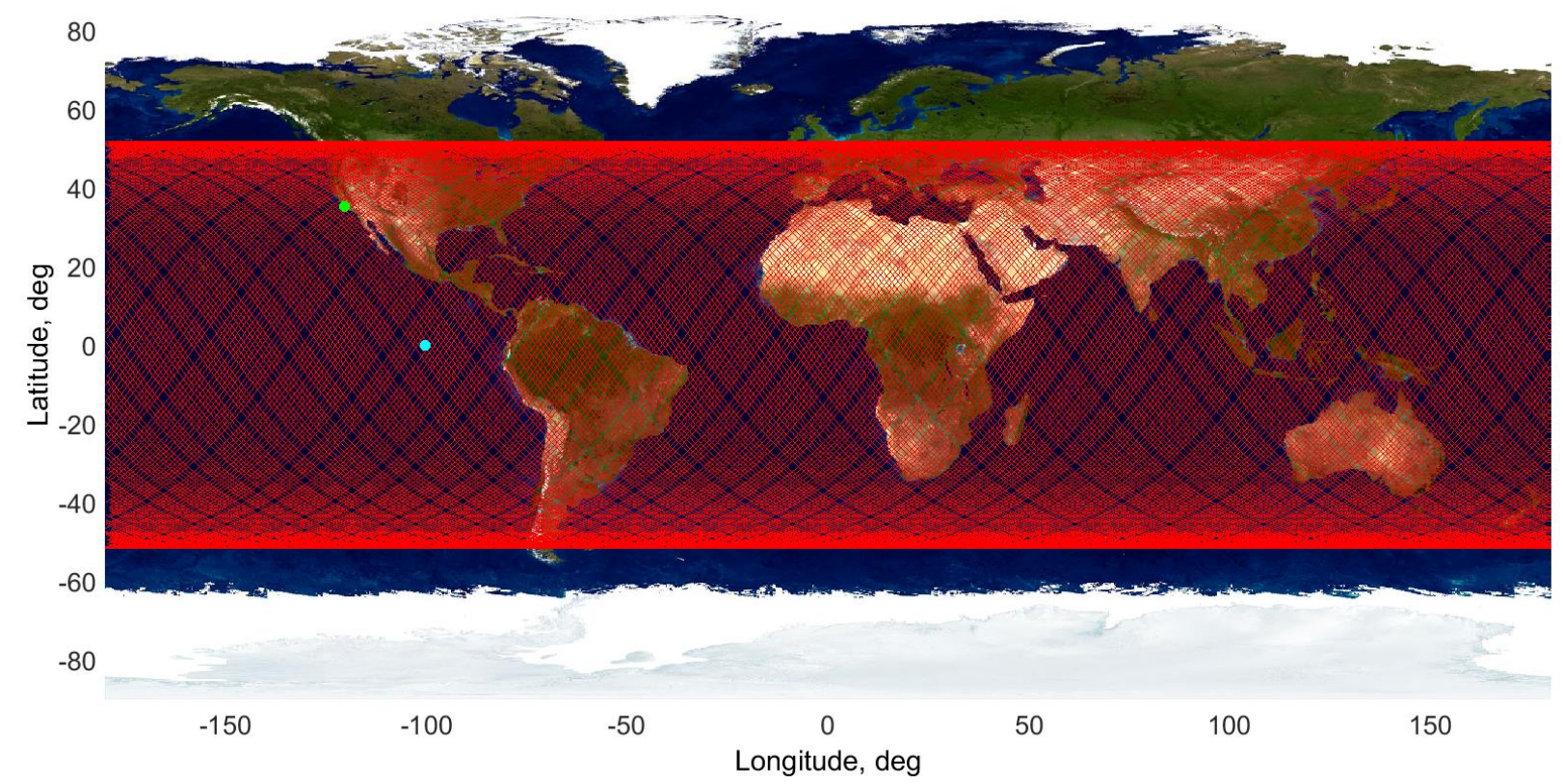

Figure 7. Ground track produced by numerical simulator for the case of a non-maneuvering satellite. The blue dot marks the position of the satellite at the beginning of the simulation. The green dot marks the position of the satellite at the end of the simulation.

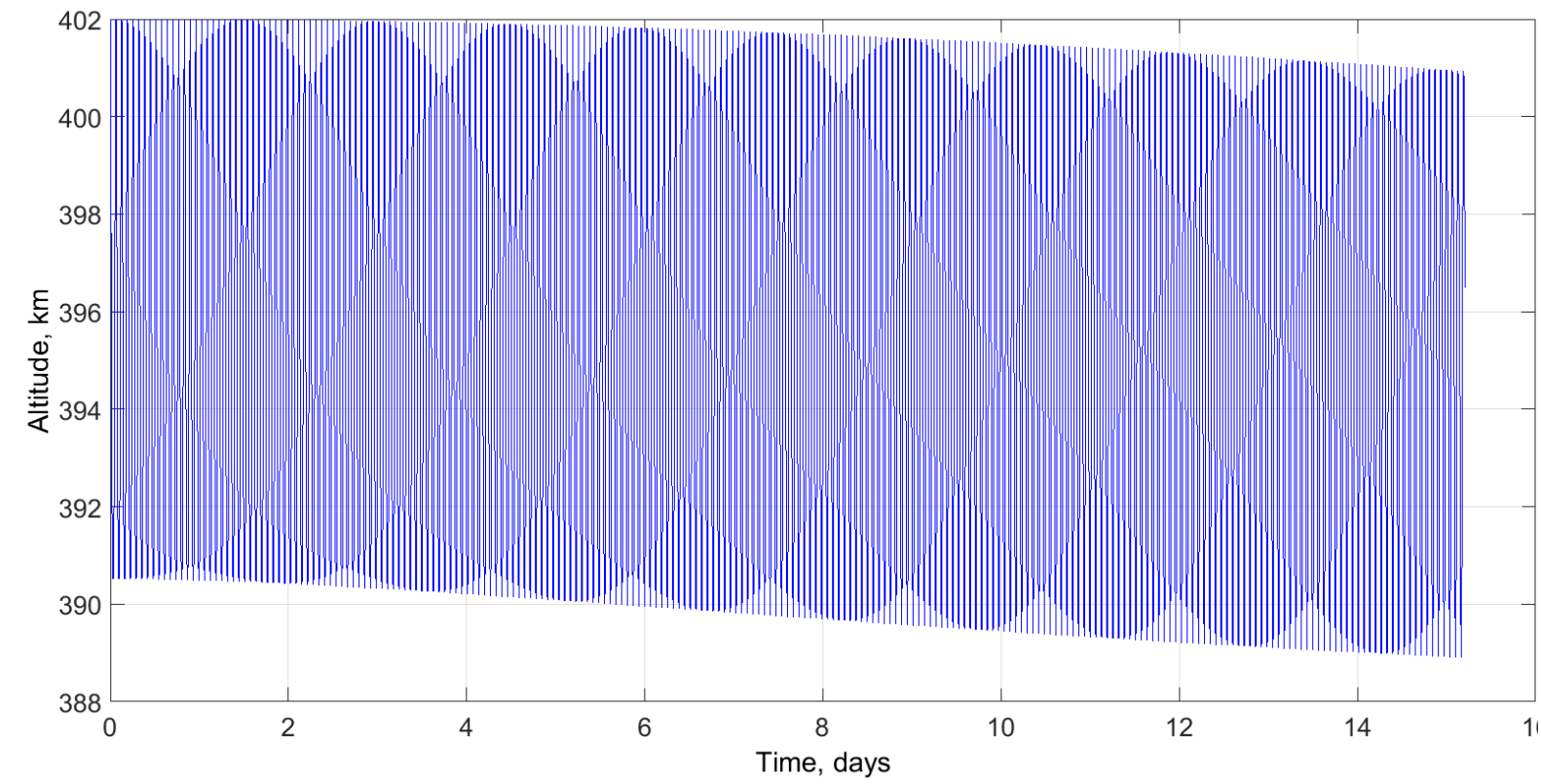

Figure 8. Altitude of the non-maneuvering satellite as calculated by the numerical simulation. 

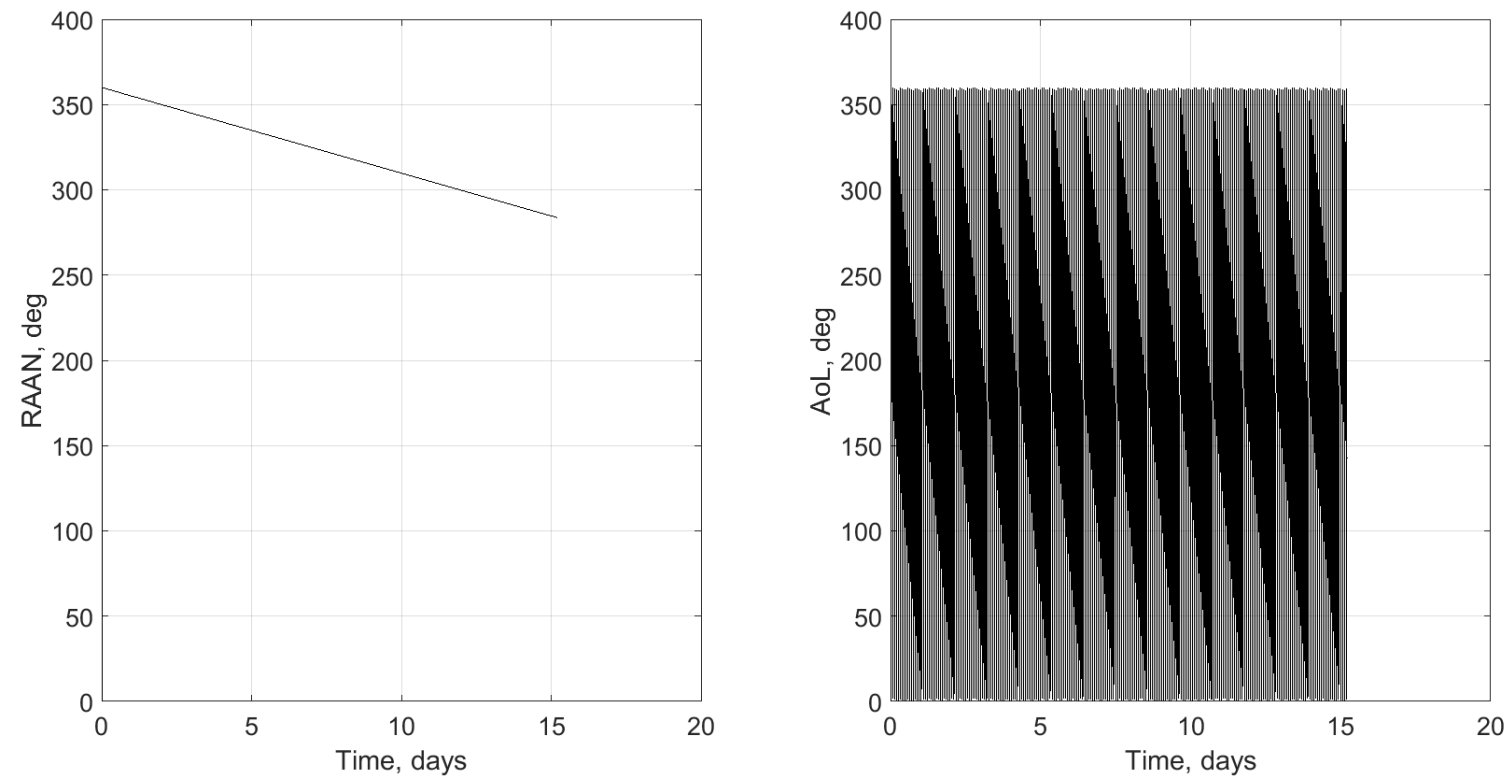

Figure 9. RAAN and AoL of the non-maneuvering satellite as calculated by the numerical simulation.

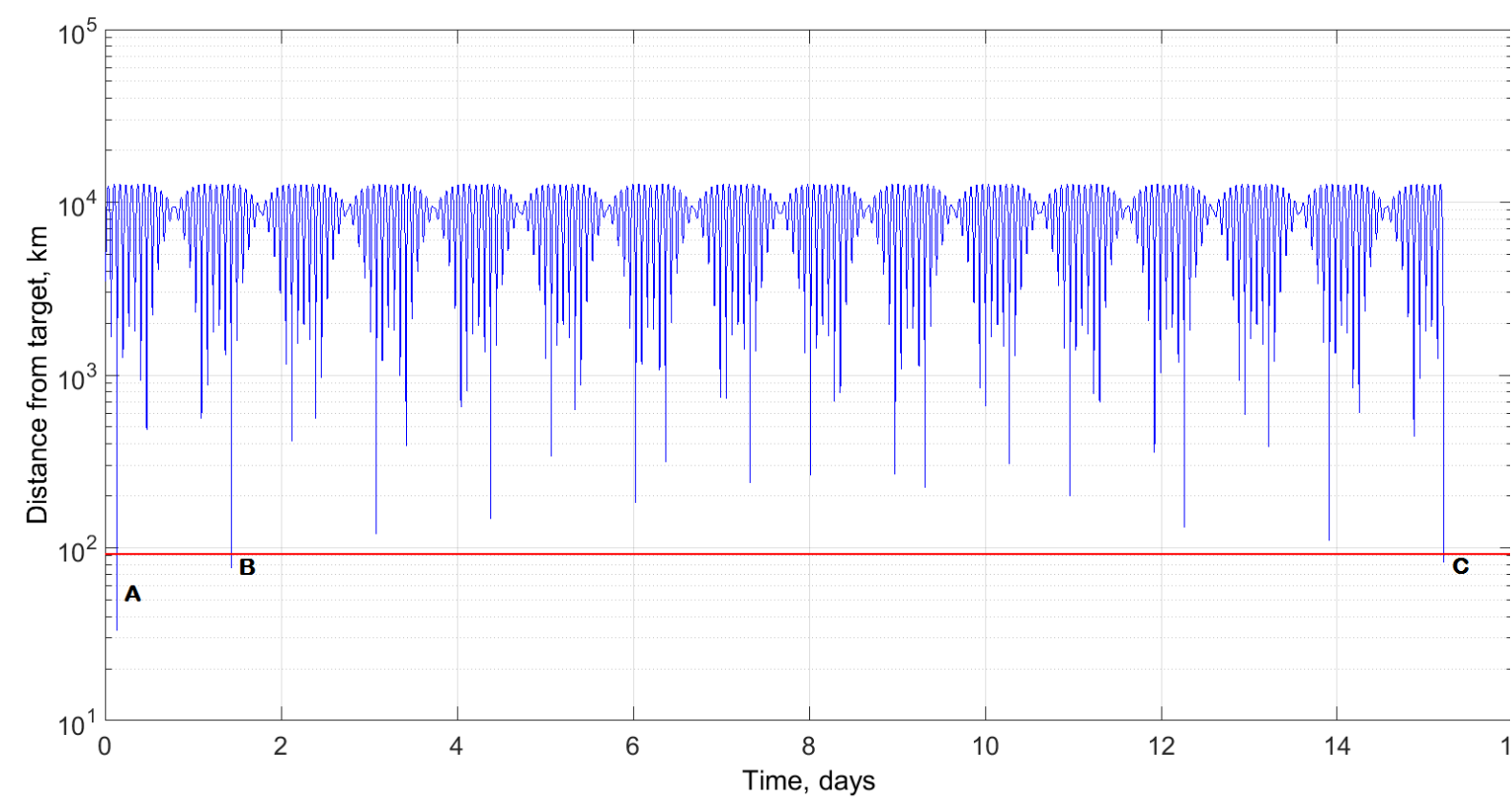

Figure 10. Distance from the SSP to the POI as measured along the surface of the Earth, calculated by the numerical simulation for the non-maneuvering satellite. The red line indicates the distance at which the POI will be in view of the satellite, assuming a swath width of $184 \mathrm{~km}$. The points marked $A, B$ and $C$ indicate the times at which the POI is in view and correspond to the passes indicated in Fig. 6.

\section{B. Validation of the method for a maneuvering satellite}

1. Analytical Method

The analytical method described above can now be used to attempt to reduce the time of successive overflights of Los Angeles. As identified in section IV.A, between passes 'B' and 'C', there is a 14 day gap during which Los Angeles will not be in view of the satellite. Taking the position of the satellite at pass $\mathrm{B}$ as the starting point, a maneuver in which the satellite lowers its altitude, drifts for a time, and then returns to its initial altitude can be analytically described for a given $\Delta \mathrm{V}_{\text {total }}$. For this validation case, $100 \mathrm{~m} / \mathrm{s} \Delta \mathrm{V}_{\text {total }}$ is used. Although the times for the two thrusting phases are fixed as functions of the maneuver $\Delta \mathrm{V}_{\text {total }}$, the drift time can 
be varied to ensure a pass over the target location at the end of the maneuver. Figure 11 shows the longitude of all passes which would finish over the target latitude, plotted against the number of revolutions for the first 100 possible upwards and downwards passes of the target latitude. Again, the grey horizontal line indicates the target longitude.

Figure 12 shows for the first 300 possible upwards and downwards passes, the distance between the SSP and the POI. The red line again indicates the point at which the POI becomes visible to the satellite. Looking at Fig. 12 the first possible pass over Los Angeles is an upwards pass after 41 revolutions, marked 'A+'. The time of this pass is 2.63 days from the start of the maneuver, shortening the flyover time of Los Angeles by more than 11 days, compared with the case where no maneuver is performed.

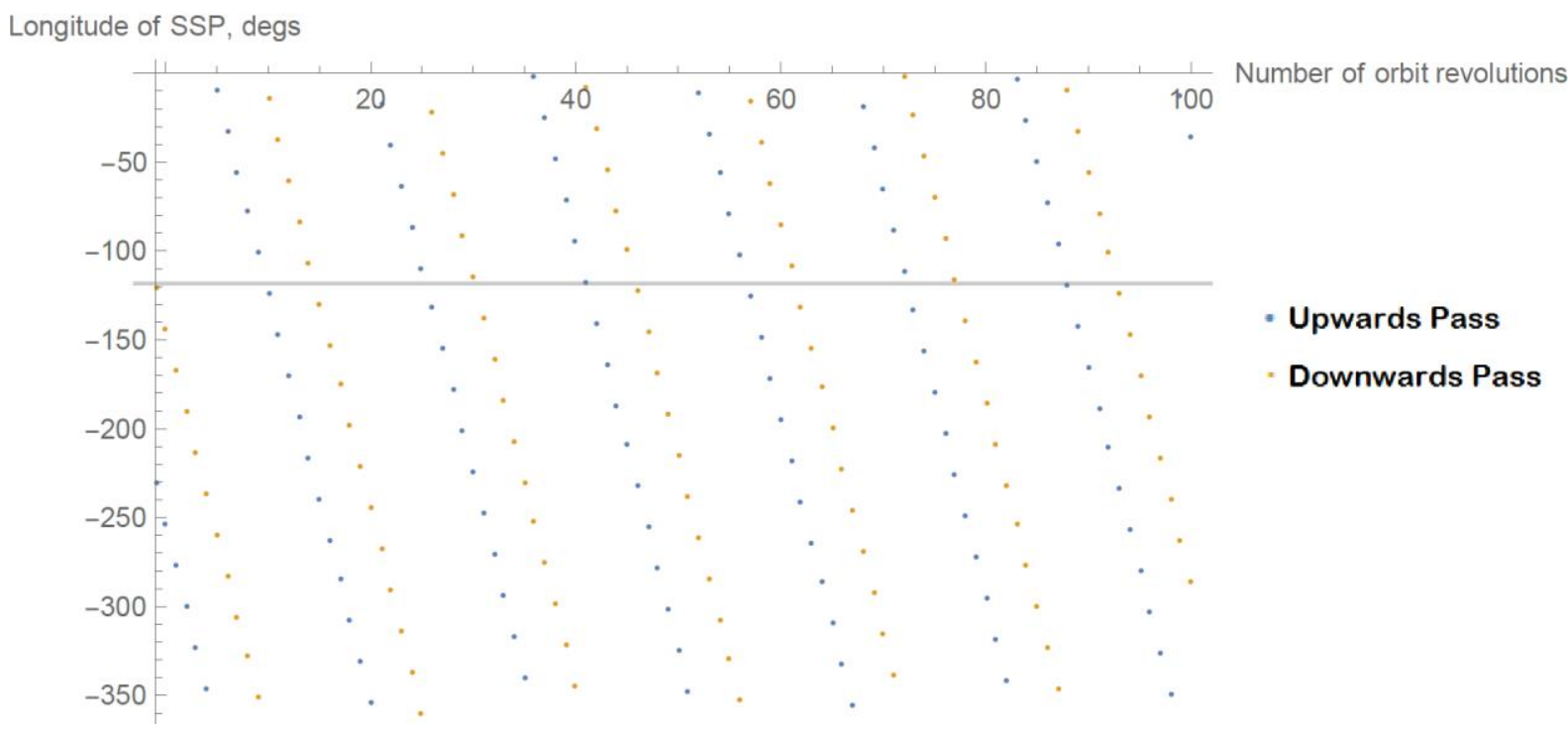

Figure 11. The longitude of the SSP for the first possible 100 passes over the target latitude with a maneuvering satellite. Blue dots mark an upwards pass and orange dots mark a downwards pass. The horizontal grey line indicates the target longitide.

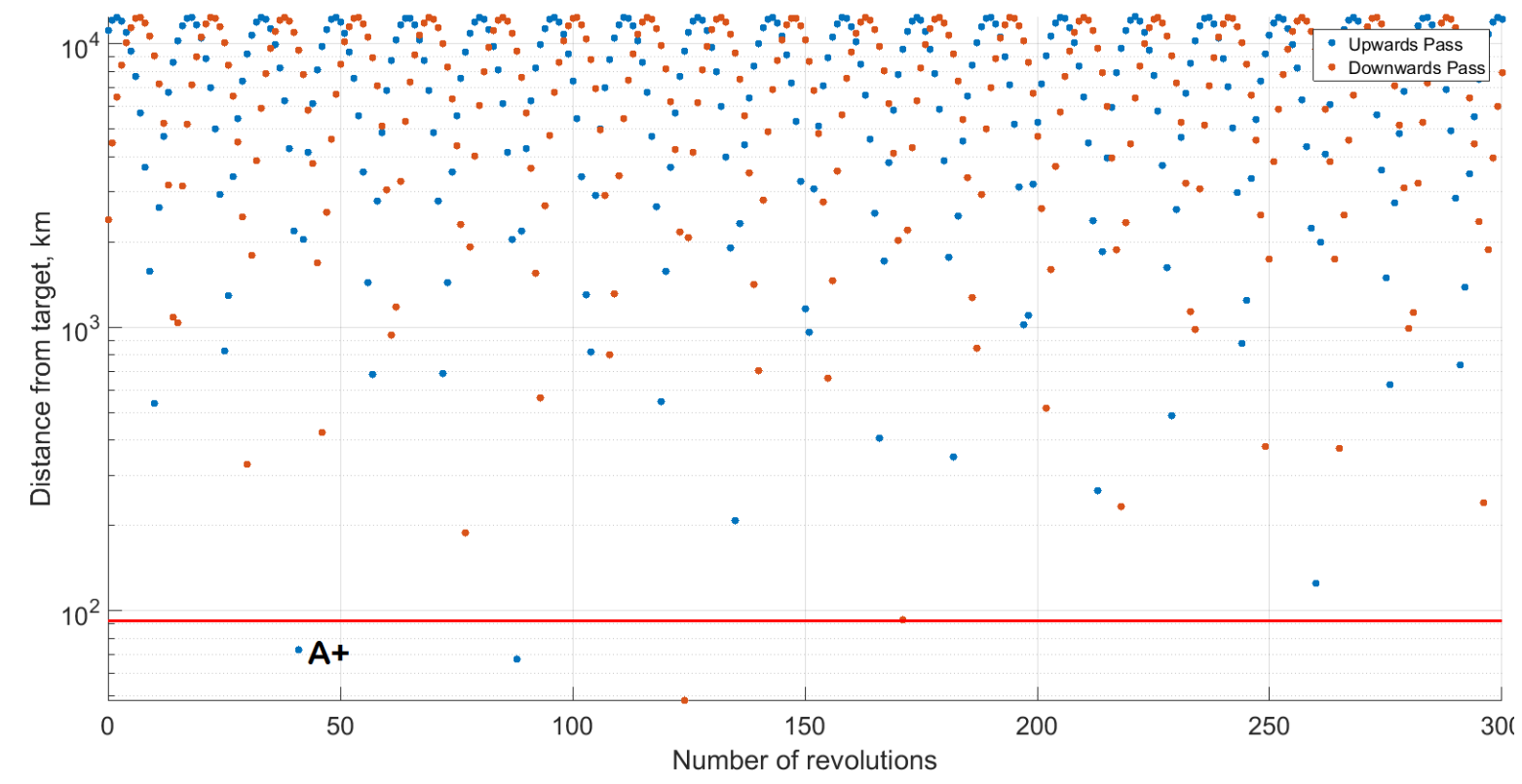

Figure 12. The distance from the SSP to the POI, plotted for the first 300 possible flyovers of the target latitude for a maneuvering satellite. Blue dots mark an upwards pass and red dots mark a downwards pass. The horizontal red line indicates the distance below which the POI will be visible. A+ marks the first possible pass over the POI. 


\section{Numerical Simulation}

Using the numerical simulator, the satellite orbit is propagated from epoch in order to validate the scenario proposed by the analytical method. In this case the initial orbit is propagated forward for 1.43 days to pass ' $\mathrm{B}$ ', at which point a constant acceleration is applied to lower the altitude of the satellite for $\frac{\Delta \mathrm{V}_{\text {total }}}{2|A|}$ seconds, corresponding to 0.58 days. The satellite orbit is then propagated for 1.48 days at the lower altitude, as calculated from the analytical maneuver, before applying a constant acceleration again for 0.58 days to return to the initial orbit altitude.

Figure 13 shows the ground track produced by the numerical propagator for this scenario. The blue dot shows the satellite position at the beginning of the simulation and the green dot shows the position of the satellite over Los Angeles at the end of the simulation. Compared to Fig. 7 which shows the ground track for a non-maneuvering satellite, Fig. 13 shows much fewer orbit revolutions, because the target is reached in a significantly shorter time. Figure 14 shows the altitude of the satellite throughout the simulation confirming that the thrust control law applied to the numerical simulation matches the maneuver defined by the analytical method. The analytical method predicts the altitude lowered to $314 \mathrm{~km}$ by the end of the first thrusting period, which matches the maneuver produced by the numerical simulator closely. Figure 15 shows the change in RAAN and AoL of the satellite throughout the simulation. From this it is clear that lowering the altitude has a limited effect on the RAAN over such a short time period, however on closer inspection it can be seen that there is a marked change to the rate of change of AoL post-maneuver, and it is this along track adjustment in positioning that allows the ground target to be passed over in a shorter time than would otherwise be possible. Figure 16 shows the distance of the satellite sub-satellite point from the target POI calculated using the haversine formula at each time step. Again, the red line indicates the distance at which the POI will be visible to the satellite. The first two peaks correspond to passes ' $\mathrm{A}$ ' and 'B', before the maneuver begins. The third peak corresponds to pass ' $\mathrm{A}+$ ' at the end of the maneuver. Again, even with the inclusion of the maneuver, the latitude and longitude of the sub-satellite point calculated using the analytical method match the numerical simulation to within $\pm 1^{\circ}$.

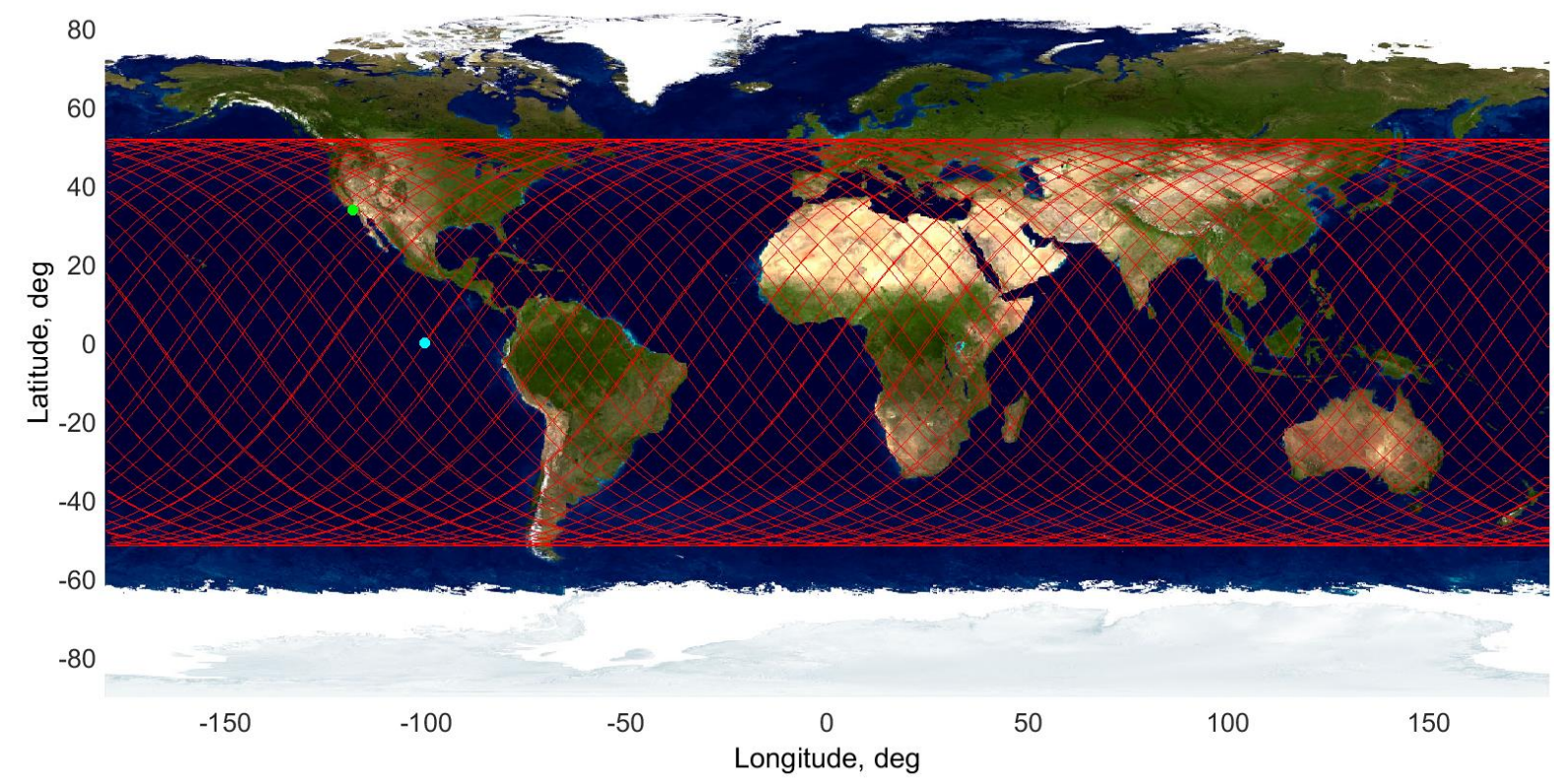

Figure 13. Ground track produced by numerical simulator for the case of a maneuvering satellite. The blue dot marks the position of the satellite at the beginning of the simulation. The green dot marks the position of the satellite at the end of the simulation. 


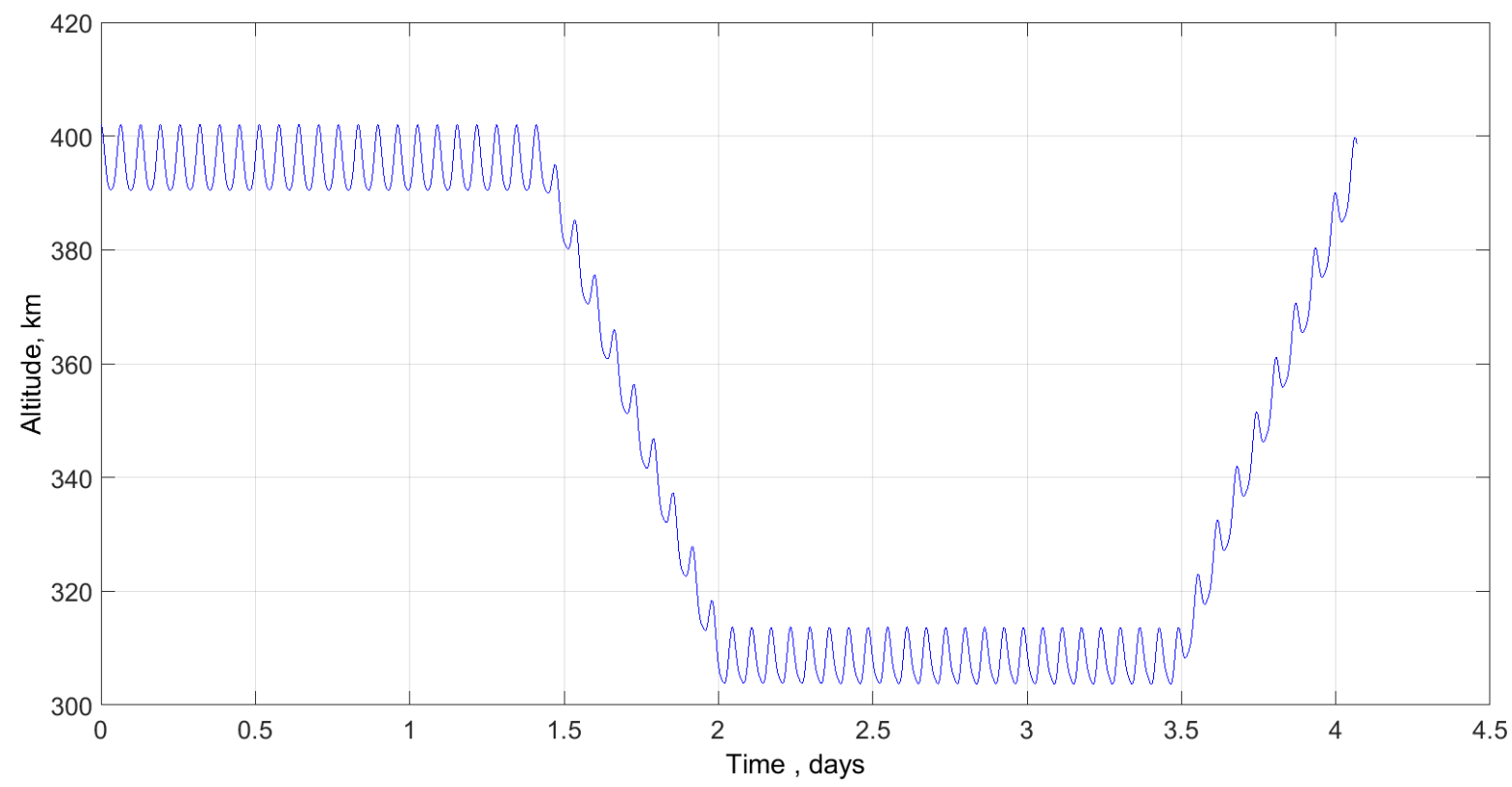

Figure 14. Altitude of the maneuvering satellite as calculated by the numerical simulation.
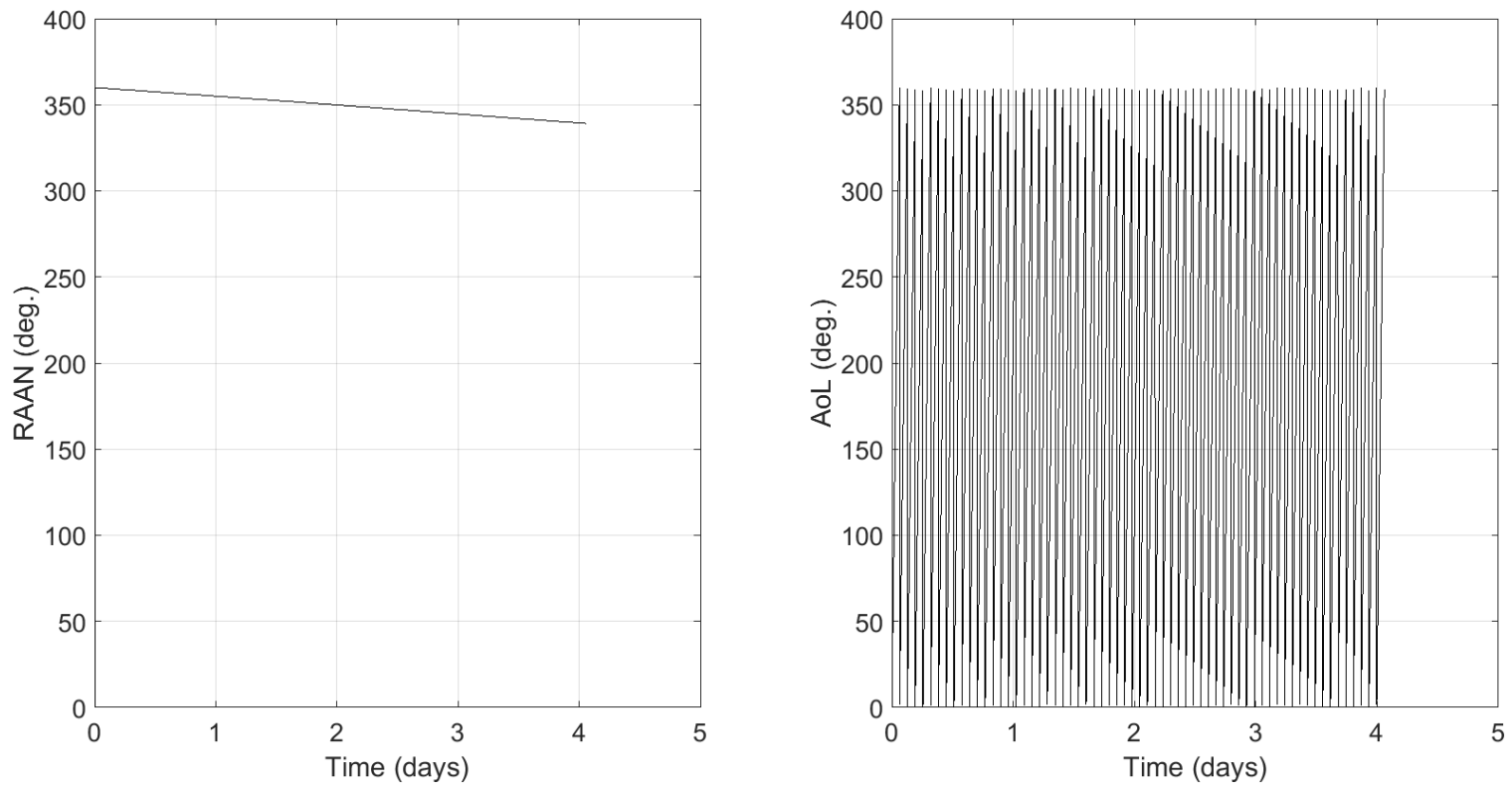

Figure 15. RAAN and AoL of the maneuvering satellite as calculated by the numerical simulation. 


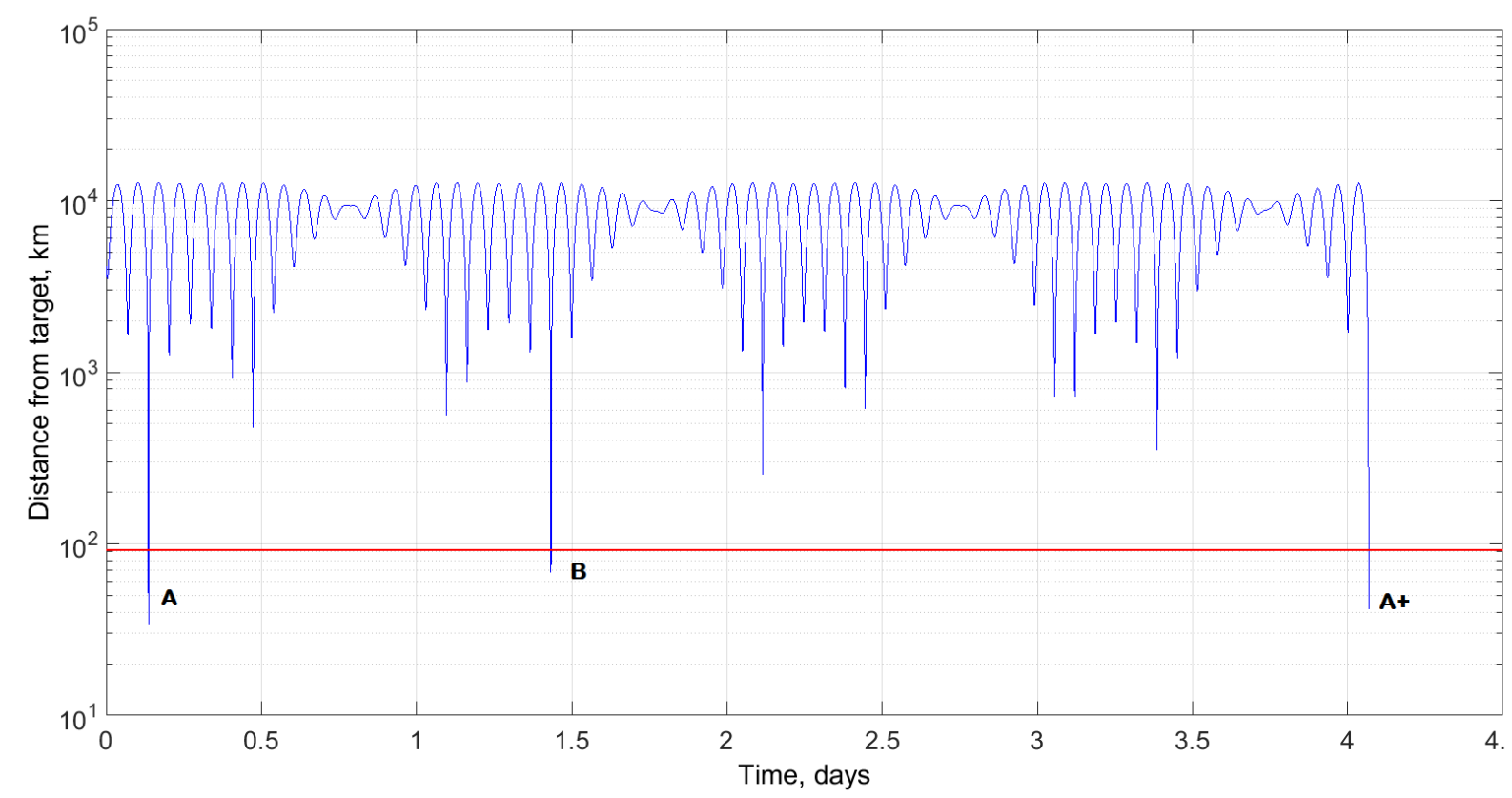

Figure 16. Distance from the SSP to the POI as measured along the surface of the Earth, calculated by the numerical simulation for the maneuvering satellite. The red line indicates the distance at which the POI will be in view of the satellite, assuming a swath width of $184 \mathrm{~km}$. The points marked $A, B$ and A+ indicate the times at which the POI is in view and correspond to the passes indicated in Fig. 6 and Fig. 12.

\section{Case Study: Rapid Return Mission to Los Angeles}

A case study is performed considering a satellite on the same orbit as the ISS which requires a rapid revisit of Los Angeles. As shown in section IV, if no maneuvers are performed there is a 14 day gap between flyovers of Los Angeles at passes ' $\mathrm{B}$ ' and ' $\mathrm{C}$ '. It has already been shown that a maneuver requiring a total $\Delta \mathrm{V}_{\text {total }}$ of $100 \mathrm{~m} / \mathrm{s}$ can reduce the time between flyovers to just 2.63 days. The same scenario is investigated here for a range of $\Delta V_{\text {total }}$ values from $0 \mathrm{~m} / \mathrm{s}$ to $200 \mathrm{~m} / \mathrm{s}$ in steps of $1 \mathrm{~m} / \mathrm{s}$. Figure 17 shows the shortest possible time in which a pass can be made over Los Angeles using this method, for each $\Delta V_{\text {total }}$ value. Time here is measured from the beginning of the maneuver, that is, from pass ' $\mathrm{B}$ '. The red line indicates the limit of what is physically achievable with the propulsion system. As an assumption of continuous thrust is made, any solutions which fall below this red line require more time to achieve the required thrust than is allotted for the maneuver.

It is clear from these graphs that the solution set is non-continuous and does not follow an obvious pattern. Increasing the $\Delta \mathrm{V}_{\text {total }}$ used for the maneuver will not necessarily give a faster flyover time of the target. Analysis of the results show that the minimum achievable flyover time for a $\Delta \mathrm{V}_{\text {total }}<200 \mathrm{~m} / \mathrm{s}$ is 1.97 days, or 47.19 hours, achieved with a $\Delta \mathrm{V}_{\text {total }}$ of $63 \mathrm{~m} / \mathrm{s}$. However, it is of note that a very similar time of flyover can be achieved using a lower $\Delta \mathrm{V}_{\text {total }}$. For example, a flyover time of 47.24 hours is achievable with $55 \mathrm{~m} / \mathrm{s} \Delta \mathrm{V}_{\text {total }}$, and with $43 \mathrm{~m} / \mathrm{s} \Delta \mathrm{V}_{\text {total }}$ a flyover time of 47.32 hours is possible. This is obvious from the solutions shown in Fig. 17, however as the solution space is discontinuous such insights may be difficult to gain through numerical methods alone. 


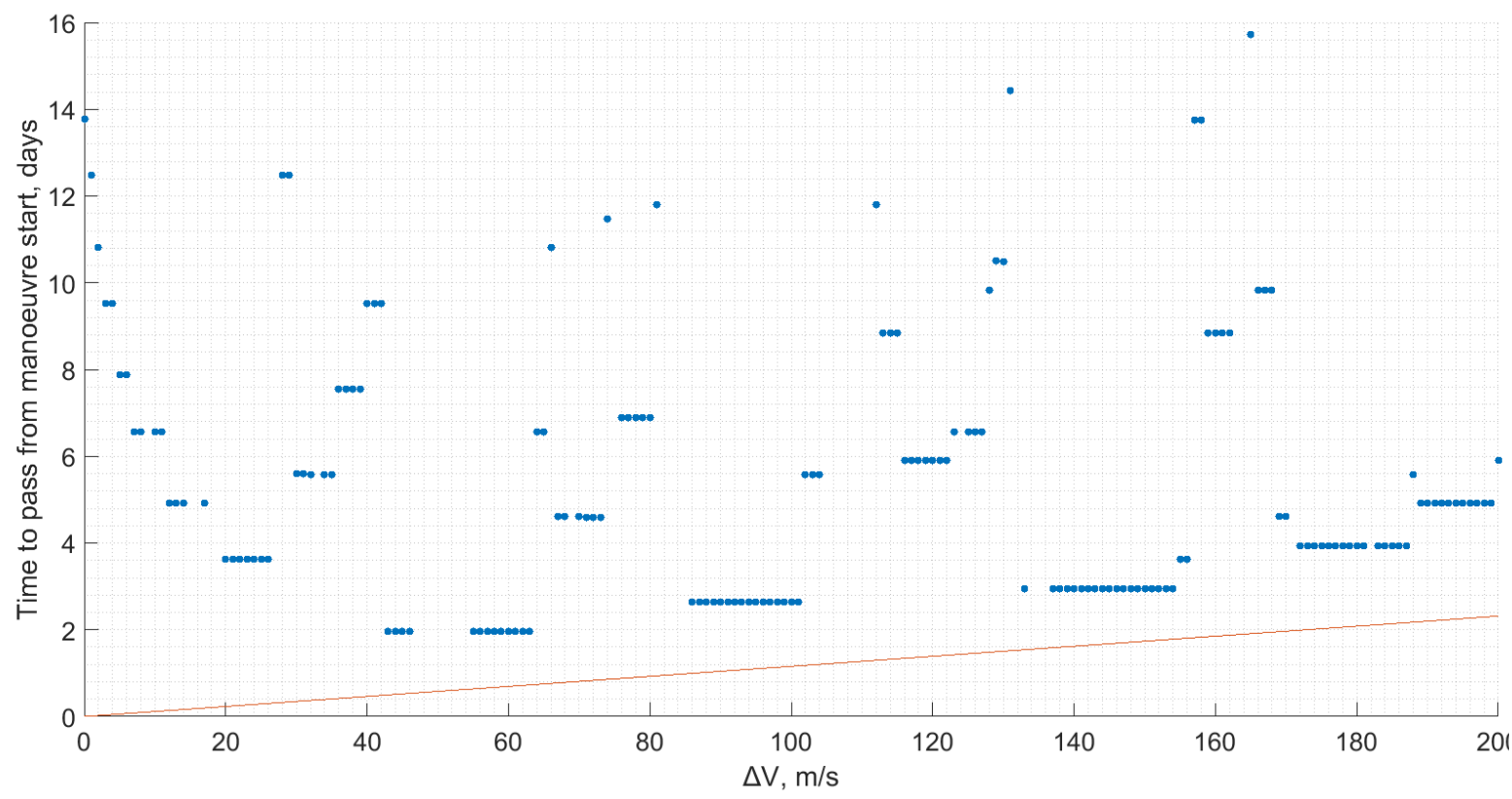

Figure 17. Time of the first possible pass over the target POI for a given $\Delta \mathbf{V}_{\text {total }}$. Solutions below the red line would not be viable as the required thrust for the maneuver could not be achieved in the given time.

\section{Conclusion}

The analytical method presented here was found to predict the time of flyover of a ground target for a maneuvering or non-maneuvering satellite with sufficient accuracy when compared with a numerical simulation, in spite of its simplifications. This analytical solution can be used to gain an insight into what is shown to be an extremely complex solution space. Such an overview of the solution space allows a greater understanding of the available maneuver options, which could aid in the decision making process of satellite operators or mission designers. The analytical method is inherently fast to run, and thus numerous possible options can be rapidly assessed. This allows a number of alternate possibilities to be assessed and compared and could be used, for example, to select the best possible satellite from a constellation to perform a maneuver, or to determine the most viable targets for overflight by a given satellite. The overview of the solution space can be used to inform trade-offs based on the possible flyover times and the $\Delta \mathrm{V}_{\text {total }}$ available by showing a range of possible solutions. It can also be used to identify possible times of flyover, allowing for the illumination conditions of the site to be considered in the trade-off.

\section{Acknowledgments}

C. N. McGrath thanks the EPSRC for their continuing financial support of her research, and her attendance at the AIAA SPACE Forum and Exhibition 2016.

\section{References}

${ }^{1}$ H. J. Kramer, Observation of the Earth and its Environment: Survey of Missions and Sensors: Springer Science \& Business Media, 2012.

${ }^{2}$ P. K. Rao, S. J. Holmes, R. K. Anderson, J. S. Winston, and P. E. Lehr, "Weather satellites: Systems, data, and environmental applications," 1990.

${ }^{3}$ J. Schulz, P. Albert, H.-D. Behr, D. Caprion, H. Deneke, S. Dewitte, et al., "Operational climate monitoring from space: the EUMETSAT Satellite Application Facility on Climate Monitoring (CM-SAF)," Atmospheric Chemistry \& Physics, vol. 9, 2009.

${ }^{4}$ F. J. Wentz and M. Schabel, "Precise climate monitoring using complementary satellite data sets," Nature, vol. 403, pp. 414-416, 2000

${ }^{5}$ W. M. Smart, Text-book on spherical astronomy: CUP Archive, 1947.

${ }^{6} \mathrm{~S}$. Voigt, T. Kemper, T. Riedlinger, R. Kiefl, K. Scholte, and H. Mehl, "Satellite image analysis for disaster and crisismanagement support," IEEE transactions on geoscience and remote sensing, vol. 45, pp. 1520-1528, 2007. 

${ }^{7} \mathrm{~F}$. P

${ }^{7}$ F. Pranajaya, R. Zee, J. Cain, and R. Kolacz, "Nanosatellite Tracking Ships: From Concept to Launch in 7 Months,"

${ }^{8}$ S. Kenyon, "Designing for Cost Effectiveness Results in Responsiveness: Demonstrating the SSTL X-Series," presented at the Reinventing Space Conference, London, UK, 2014.

${ }^{9}$ M. Guelman and A. Kogan, "Electric propulsion for remote sensing from low orbits," Journal of guidance, control, and dynamics, vol. 22, pp. 313-321, 1999.

${ }^{10}$ I. Jean and I. J. de Lafontaine, "Autonomous guidance and control of an Earth observation satellite using low thrust," Advances in the Astronautical Sciences, vol. 116, pp. 1829-1844, 2003.

${ }^{11} \mathrm{O}$. Abdelkhalik and A. Gad, "Optimization of space orbits design for Earth orbiting missions," Acta Astronautica, vol. 68, pp. 1307-1317, 2011.

${ }^{12}$ O. Abdelkhalik, "Initial orbit design from ground track points," Journal of Spacecraft and Rockets, vol. 47, pp. 202205, 2010.

${ }^{13}$ R.-D. Kim, O.-C. Jung, and H. Bang, "A computational approach to reduce the revisit time using a genetic algorithm," in Control, Automation and Systems, 2007. ICCAS'07. International Conference on, 2007, pp. 184-189.

${ }^{14}$ O. L. De Weck, U. Scialom, and A. Siddiqi, "Optimal reconfiguration of satellite constellations with the auction algorithm," Acta Astronautica, vol. 62, pp. 112-130, 2008.

${ }^{15}$ K.-J. Zhu, J.-F. Li, and H.-X. Baoyin, "Satellite scheduling considering maximum observation coverage time and minimum orbital transfer fuel cost," Acta Astronautica, vol. 66, pp. 220-229, 2010.

${ }^{16} \mathrm{G}$. Zhang, X. Cao, and D. Mortari, "Analytical approximate solutions to ground track adjustment for responsive space," IEEE Transactions on Aerospace and Electronic Systems, vol. 52, pp. 1366-1383, 2016.

${ }^{17}$ G. Zhang and J. Sheng, "Impulsive Ground-Track Adjustment for Assigned Final Orbit," Journal of Spacecraft and Rockets, pp. 1-11, 2016.

${ }^{18}$ T. C. Co and J. T. Black, "Responsiveness in Low Orbits Using Electric Propulsion," Journal of Spacecraft and Rockets, vol. 51, pp. 938-945, 2014.

${ }^{19}$ T. C. Co, C. Zagaris, and J. T. Black, "Responsive Satellites Through Ground Track Manipulation Using Existing Technology," Journal of Spacecraft and Rockets, vol. 50, pp. 206-216, 2013.

${ }^{20}$ T. C. Co, "Operationally responsive spacecraft using electric propulsion," DTIC Document2012.

${ }^{21}$ C. McGrath and M. Macdonald, "Design of a Reconfigurable Satellite Constellation," presented at the 66th International Astronautical Congress, Jerusalem, Israel, 2015.

${ }^{22}$ C. McGrath, E. Kerr, and M. Macdonald, "An analytical low-cost deployment strategy for satellite constellations " presented at the 13th Reinventing Space Conference, Oxford, 2015.

${ }^{23}$ D. A. Vallado and W. D. McClain, Fundamentals of astrodynamics and applications vol. 12: Springer Science \& Business Media, 2001

${ }^{24}$ S. Q. Kidder and T. H. V. Haar, Satellite meteorology: an introduction: Gulf Professional Publishing, 1995.

${ }^{25} \mathrm{M}$. Walker, B. Ireland, and J. Owens, "A set modified equinoctial orbit elements," Celestial mechanics, vol. 36, pp. 409-419, 1985.

${ }^{26}$ J. R. Dormand and P. J. Prince, "A family of embedded Runge-Kutta formulae," Journal of computational and applied mathematics, vol. 6, pp. 19-26, 1980. 